\title{
Large-Amplitude Long-Wave Instability of a Supersonic Shear Layer
}

\author{
By A. F. Messiter
}

\begin{abstract}
For sufficiently high Mach numbers, small disturbances on a supersonic vortex sheet are known to grow in amplitude because of slow nonlinear wave steepening. Under the same external conditions, linear theory predicts slow growth of long-wave disturbances to a thin supersonic shear layer. An asymptotic formulation that adds nonzero shear-layer thickness to the weakly nonlinear formulation for a vortex sheet is given here. Spatial evolution is considered for a spatially periodic disturbance having amplitude of the same order, in Reynolds number, as the shear-layer thickness. A quasi-equilibrium inviscid nonlinear critical layer is found, with effects of diffusion and slow growth appearing through a nonsecularity condition. Other limiting cases are also considered, in an attempt to determine a relationship between the vortex-sheet limit and the long-wave limit for a thin shear layer; there appear to be three special limits, corresponding to disturbances of different amplitudes at different locations along the shear layer.
\end{abstract}

\section{Introduction}

According to the linear theory, a vortex sheet in supersonic flow is neutrally stable if the Mach number is large enough. For example, if the temperature is the same on both sides of the sheet, neutral stability is predicted when the

Address for correspondence: Department of Aerospace Engineering, University of Michigan, Ann Arbor, MI 48109-2118. 
difference in Mach numbers on the two sides is greater than $2 \sqrt{2}$; otherwise disturbances grow exponentially with time. Thus if the velocities are equal and opposite above and below, the Mach number in each part of the flow must be greater than $\sqrt{2}$ to avoid instability. For higher Mach numbers, there is a stationary mode in this coordinate system, as well as a pair of propagating modes that move at speeds depending on the Mach number. The definitive presentation of these results was given by Miles [1], who gives additional references.

Artola and Majda [2] have considered the nonlinear growth of a disturbance to a vortex sheet that is caused by an incident sound wave. For incidence angles such that the component of wave number along the sheet equals the wave number of one of the neutrally stable linear modes, the nonlinearity causes a wave steepening that leads to the formation of weak shock waves and centered expansions on opposite sides of the sheet. The slow evolution of disturbances to the vortex sheet occurs on a scale that is large in comparison with the wavelength by a factor proportional to the ratio of the wavelength to the amplitude of the disturbance. Similar formation of nonlinear "kink" modes for specific incidence angles has been observed in numerical calculations by Pedelty and Woodward [3] and Bassett and Woodward [4].

For a shear layer of nonzero thickness, linear theory leads to an eigenvalue problem whose solution gives the complex wave speed. Blumen et al. [5] have shown that an expansion for wavelengths large in comparison with the thickness gives in the limit the same neutral wave speeds as for a vortex sheet, subject to the same condition on the Mach number. The wave speed, however, has a small imaginary part, implying a slow exponential growth of small disturbances in a time or distance larger than the period or wavelength by a factor proportional to the ratio of wavelength to shear-layer thickness.

Balsa [6, 7] has observed that nonlinearity influences this shear-layer instability when the ratio of disturbance amplitude to shear-layer thickness becomes of the same order as the ratio of shear-layer thickness to disturbance wavelength. In a long-wave limit when these two ratios are of the same small order, the asymptotic description of slow changes in the flow requires matching of solutions for the inviscid external flow, for the main part of the shear layer, and for the critical layer where the flow velocity is close to the wave speed. The result is a pair of partial differential equations describing a nonequilibrium, nonlinear, viscous critical layer, coupled with a condition for the velocity jump across the critical layer in the form of an evolution equation for the amplitude. Numerical solutions show the beginning of vortex rollup within the critical layer on a spatial scale larger than a wavelength by a factor proportional to the ratio of wavelength to thickness.

In the case studied here, the disturbance amplitude and shear-layer thickness are taken to be of the same order. The formulation can be thought 
of as extending the formulation for a vortex sheet by incorporating the effects of nonzero shear-layer thickness. That is, a limiting case is chosen such that the thin shear layer experiences slow spatial growth of small long-wave disturbances that are described in terms of a quasi-equilibrium nonlinear critical layer and an external flow with nonlinear wave steepening. The problem statement is outlined in Section 2. Solutions for the external flow, for the main part of the shear layer, and for the critical layer are given in Sections 3, 4, and 5; a preliminary version of these results was given in [8]. Some other limiting cases are discussed in Section 6, corresponding to different amplitudes and/or locations along the shear layer. The present formulation and that of $[6,7]$ are seen to represent different special limits of the equations, and a third special limit can also be identified.

\section{Problem statement}

A thin laminar shear layer between two parallel streams is subjected to a two-dimensional disturbance of small amplitude, traveling in the direction of shear-layer growth, with parameters chosen such that the relative disturbance speed is supersonic both above and below the shear layer and such that the growth rate of the disturbance is small. Asymptotic solutions are sought in each of three flow regions: the external inviscid flow, the main part of the thin shear layer, and the still thinner critical layer where the flow speed and disturbance speed are nearly equal.

There are four independent length scales: a viscous length (based on a reference value of flow velocity), a representative shear-layer thickness, a disturbance wavelength (or spatial period), and a typical transverse displacement of the shear layer. Three nondimensional ratios can then be defined, for example, as follows: $\varepsilon$ is the ratio of disturbance amplitude to wavelength; $\delta$ is the ratio of shear-layer thickness to disturbance wavelength; and $\mathrm{Re}$ is a Reynolds number based on the wavelength and on the reference values chosen for the velocity and kinematic viscosity, say the values in the undisturbed upper stream. Asymptotic solutions for the perturbed flow variables are then sought in the limit as the three parameters $\varepsilon, \delta$, and $1 / \operatorname{Re}$ approach zero, in terms of suitably defined coordinates corresponding to each of the three flow regions. The relative sizes specified for the three small parameters will depend on the choice of a particular limiting case to be studied. Here the thickness and amplitude are taken to be of the same order, so that

$$
\varepsilon=O(\delta) .
$$


A relation between the orders of $\delta$ and $1 / \mathrm{Re}$ is chosen in Section 5 so as to provide a balance between viscous effects and slow distortion of the flow.

All variables to be introduced are nondimensional. Lengths are made nondimensional with respect to the wavelength (or spatial period) of the disturbance. Velocities and thermodynamic variables are made nondimensional with respect to some convenient set of reference values, say the values of these quantities in the undisturbed upper stream.

The coordinates $x$ and $y$ lie along and normal to the undisturbed stream above the shear layer, with origin at the origin of the shear layer. Changes occurring on scales $O(1 / \varepsilon)$ are represented in terms of "slow" variables $\bar{x}, \bar{y}$, and $\bar{t}$ chosen to agree with the scales for slow nonlinear wave steepening in the external inviscid supersonic flow:

$$
\bar{x}=\varepsilon(x-L), \quad \bar{y}=\varepsilon y, \quad \bar{t}=\varepsilon t,
$$

where $L$ is a nondimensional downstream distance characterizing the region where nonlinear disturbance growth is to be studied; the use of analogous variables in the unsteady one-dimensional case is shown in [9]. The relationship between the orders of $\delta$ and $1 / \operatorname{Re}$ chosen in Section 5 is such that the slow changes do occur on a scale larger than a wavelength but smaller than the length of the shear layer. The slow spatial growth of the shear layer due to viscous diffusion introduces only higher-order changes on the scale considered, since the flow is nearly parallel on the scale given by (2.2). Moreover, the spatial extent of the assumed disturbance is taken to be small in comparison with the distance from the origin of the shear layer, but large in comparison with the scale for the slow variable $\bar{x}$; solutions are sought on the scale of $\bar{x}$ but not on the still larger scale measuring the extent of the disturbance. Temporal or spatial instability can be studied; spatial instability will be considered here, since the flow is expected to be convectively unstable [10]. The dependence on $\bar{t}$ is retained only through the beginning of Section 3 and is then dropped for spatial instability.

The nondimensional velocity components in the $x$-and $y$-directions are $u$ and $v$, and $c$ is the nondimensional speed of a disturbance traveling in the positive $x$-direction. A coordinate $X$ is measured relative to this disturbance, and a coordinate $Y$ is measured from a reference location $y=A$ within the shear layer:

$$
X=x-c t, \quad Y=y-A(X, \bar{x}, \bar{t}) .
$$

The nondimensional pressure, density, temperature, and viscosity coefficient are $p, \rho, T$, and $\mu$, respectively. The Mach number $M$ is defined in terms of the reference quantities mentioned, as is the Prandtl number Pr, which is 
assumed constant. The nondimensional velocities, temperatures, and densities in the undisturbed upper and lower streams are denoted by $u=u_{ \pm}$, $T=T_{ \pm}$, and $\rho=\rho_{ \pm}$, respectively. The undisturbed pressure is taken to be the same above and below the shear layer, and it is assumed that the ratio of specific heats is likewise the same; the specific heats are taken to be constant. If the reference quantities are the values in the upper stream, then $u_{+}=T_{+}=\rho_{+}=1$ and $p_{+}=p_{-}=1$; the notation $u_{ \pm}, T_{ \pm}$, and $\rho_{ \pm}$is, however, retained for convenience. We take $u_{+}>u_{-}$and consider disturbances traveling in the direction of increasing $x$, with relative speeds supersonic both above and below. Some of the notation, including the expansion (3.5) of $A$, is indicated in Figure 1.

A shear-layer coordinate $\tilde{y}$ is defined by

$$
\tilde{y}=\frac{Y}{\delta}
$$

and the undisturbed velocity and temperature profiles are expressed in terms of this coordinate by $u=\tilde{u}_{0}(\tilde{y})$ and $T=\tilde{T}_{0}(\tilde{y})$. The displacement function $A$ introduced in (2.3) is defined such that $\tilde{y}=0$ when $\tilde{u}_{0}=c$, A critical-layer coordinate $\hat{y}$ is needed in Section 5 and is defined there by

$$
\hat{y}=\frac{\tilde{y}}{\varepsilon^{1 / 2}} \text {. }
$$

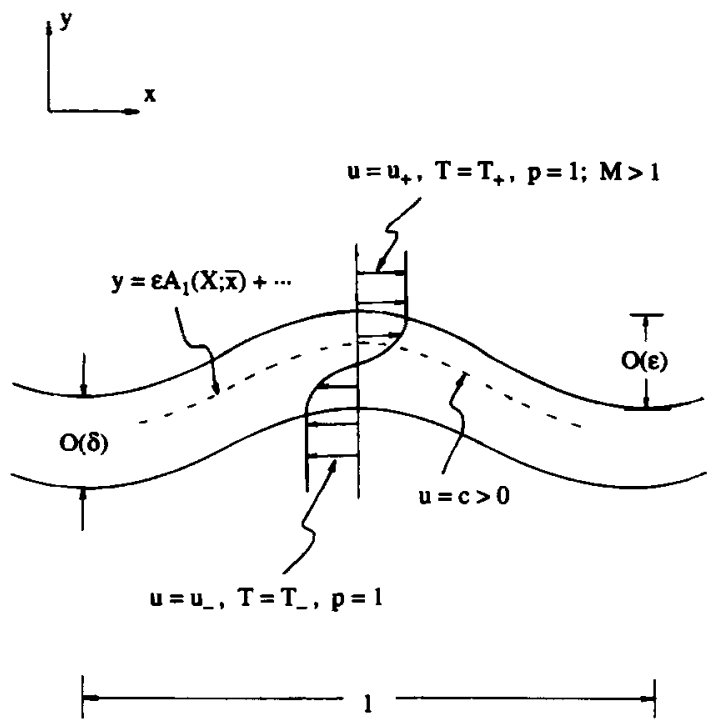

Figure 1. Disturbed shear layer with $\varepsilon=O(\delta)$; amplitude depends on $X=x-c t$ and $\bar{x}=\varepsilon x$. 
The starting point should be the full set of conservation laws, containing all the terms representing effects of viscosity and heat conduction. For brevity, many terms are omitted here because they are not required in the leading approximations for the description of an inviscid flow or a thin shear layer. The needed terms in the continuity, $x$-momentum, $y$-momentum, energy, and state equations are, at most,

$$
\begin{gathered}
\rho_{t}+(\rho u)_{x}+(\rho v)_{y}=0 \\
\rho\left(u_{t}+u u_{x}+v u_{y}\right)+\frac{1}{\gamma M^{2}} p_{x}=\frac{1}{\operatorname{Re}}\left(\mu u_{y}\right)_{y}+\cdots \\
\rho\left(v_{t}+u v_{x}+v v_{y}\right)+\frac{1}{\gamma M^{2}} p_{y}=\cdots \\
\rho\left(T_{t}+u T_{x}+v T_{y}\right)-\frac{\gamma-1}{\gamma}\left(p_{t}+u p_{x}+v p_{y}\right) \\
=\frac{1}{\operatorname{Re} \operatorname{Pr}}\left(\mu T_{y}\right)_{y}+(\gamma-1) \frac{M^{2}}{\operatorname{Re}} \mu u_{y}^{2}+\cdots \\
p=\rho T .
\end{gathered}
$$

In the external flow the right side of (2.9) is of higher order than the terms needed, and the entropy is nearly uniform:

$$
p=\left(\rho / \rho_{ \pm}\right)^{\gamma}+\cdots, \quad d p=\gamma\left(\rho / \rho_{ \pm}\right)^{\gamma-1} d\left(\rho / \rho_{ \pm}\right)+\cdots,
$$

where the upper and lower signs again refer to quantities in the undisturbed upper and lower streams respectively. Within the shear layer, dissipative effects cannot be ignored, and it is convenient to introduce a differential equation for the spanwise vorticity component

$$
\Omega=v_{x}-u_{y} .
$$

Taking the curl of the vector momentum equation, and using the continuity and state equations, again including only terms that will be needed for the thin shear layer (as can be verified later), we have

$$
\rho\left(\frac{\Omega}{\rho}\right)_{t}+\rho u\left(\frac{\Omega}{\rho}\right)_{x}+\rho v\left(\frac{\Omega}{\rho}\right)_{y}=\frac{1}{\gamma M^{2} p} T_{y} p_{x}+\frac{1}{\operatorname{Re}} \frac{1}{\rho}(\mu \Omega)_{y y}+\cdots .
$$




\section{External flow}

In the external flow the length scale in each direction is the wavelength, or spatial period. The perturbed flow variables are functions of the coordinates $X$ and $Y$ introduced in (2.3), and of the slow variables defined by (2.2), and are assumed to possess expansions of the form

$$
\begin{aligned}
u & =u_{ \pm}+\varepsilon u_{1}(X, Y, \bar{x}, \bar{y}, \bar{t})+\varepsilon^{2} u_{2}(X, Y, \bar{x}, \bar{y}, \bar{t})+\cdots \\
v & =\varepsilon v_{1}(X, Y, \bar{x}, \bar{y}, \bar{t})+\varepsilon^{2} v_{2}(X, Y, \bar{x}, \bar{y}, \bar{t})+\cdots \\
p & =1+\varepsilon p_{1}(X, Y, \bar{x}, \bar{y}, \bar{t})+\varepsilon^{2} p_{2}(X, Y, \bar{x}, \bar{y}, \bar{t})+\cdots \\
\frac{\rho}{\rho_{ \pm}} & =1+\varepsilon \rho_{1}(X, Y, \bar{x}, \bar{y}, \bar{t})+\varepsilon^{2} \rho_{2}(X, Y, \bar{x}, \bar{y}, \bar{t})+\cdots
\end{aligned}
$$

The displacement function $A$ is expanded as

$$
A=\varepsilon A_{1}(X, \bar{x}, \bar{t})+\varepsilon^{2} A_{2}(X, \bar{x}, \bar{t})+\cdots
$$

All the perturbations are expected to depend on the ratio $\varepsilon / \delta$ as well as on the variables shown, and the dependence on $\bar{t}$ is dropped later. It is in fact premature to specify the orders appearing in the second approximation before the solutions for the first approximation have been completed in all three flow regions. Once the matching of these solutions has been carried out, each of the functions $u_{2}, v_{2}, p_{2}$, and $\rho_{2}$ is seen to contain a term proportional to $\ln \varepsilon$. These terms do not play a major role in the solution; for conciseness of notation, they are not shown explicitly.

The first approximations to the continuity, $X$-momentum, and $Y$-momentum equations are found by retaining only terms of order $\varepsilon$, as follows:

$$
\begin{aligned}
& \left(u_{ \pm}-c\right) \rho_{1 X}+u_{1 X}+v_{1 Y}=0 \\
& \left(u_{ \pm}-c\right) u_{1 X}+\frac{1}{\rho_{ \pm} M^{2}} \rho_{1 X}=0 \\
& \left(u_{ \pm}-c\right) v_{1 X}+\frac{1}{\rho_{ \pm} M^{2}} \rho_{1 Y}=0
\end{aligned}
$$

Here the quantities $u_{+}-c$ and $c-u_{-}$can be thought of as the propagation speeds of a disturbance in the shear layer relative to the flow speeds in the upper and lower streams, or $u_{+}-c>0$ and $u_{-}-c<0$ are the flow speeds above and below as seen in the $X, Y$ coordinate system moving with the 
disturbance. The two quantities denoted by $M^{2} \rho_{ \pm}\left(u_{ \pm}-c\right)^{2}$ are then the squares of the relative Mach numbers based on these speeds and on $\rho_{ \pm}$. The parameters are chosen such that each of these expressions is greater than one and thus that the relative speeds are supersonic both above and below the sheet. In other words, we consider cases where

$$
u_{-}+\frac{1}{\rho_{-}^{1 / 2} M}<c<u_{+}-\frac{1}{\rho_{+}^{1 / 2} M}
$$

The equation defining $c$ is given in Section 4 and is the same as for a vortex sheet [1].

Elimination of $u_{1}$ and $v_{1}$ above leads to a wave equation for $\rho_{1}$ :

$$
\beta_{ \pm}^{2} \rho_{1 X X}-\rho_{1 Y Y}=0, \quad \beta_{ \pm}^{2}=M^{2} \rho_{ \pm}\left(u_{ \pm}-c\right)^{2}-1,
$$

where $\beta_{ \pm}^{2}>0$, as noted previously. Solutions for $\rho_{1}, u_{1}$, and $v_{1}$ that describe disturbances traveling outward from the shear layer are then found in the form

$$
\frac{v_{1}}{u_{ \pm}-c}=-\frac{\beta_{ \pm} u_{1}}{u_{ \pm}-c}=\frac{\beta_{ \pm} \rho_{1}}{\rho_{ \pm}\left(u_{ \pm}-c\right)^{2} M^{2}}=\left(F_{1 \pm}\right)_{\xi}(\xi, \bar{x}, \bar{t}, \bar{y})
$$

where $\xi$ is a coordinate that is constant along linearized outgoing characteristics:

$$
\xi=X-\beta_{ \pm} Y
$$

These are the usual results of linear supersonic aerodynamics. While $\xi$ has not been written with a subscript, it is to be understood that the definition requires $\beta_{+}$when $Y>0$ and $\beta_{-}$when $Y<0$. The notation $F_{1 \pm}$ allows the possibility that the function $F_{1+}$ for $Y>0$ might be different from the function $F_{1-}$ for $Y<0$. It is anticipated, however, that in the first approximation the streamline slopes are the same above and below the shear layer as $Y \rightarrow \pm 0$ and that each is equal to the leading term in the slope $A_{X}$ of the surface (3.5). Thus, after integration,

$$
\left.F_{1+}\right|_{Y=0}=\left.F_{1-}\right|_{Y=0}=A_{1}
$$

and therefore also $F_{1+}$ and $F_{1-}$ are the same functions of the argument $\xi$ everywhere, for $Y \neq 0$. This result is shown more clearly in Section 4, but for 
simpler notation is assumed here as well, and the replacement

$$
\left(F_{1+}\right)_{\xi}=\left(F_{1-}\right)_{\xi}=\theta
$$

is made below, where it might again be emphasized that the definition (3.12) for $\xi$ is different for $Y>0$ and $Y<0$.

The terms of order $\varepsilon^{2}$ in the continuity, $X$-momentum, and $Y$-momentum equations give the equations to be solved for the second terms in the expansions of $u, v$, and $\rho$ :

$$
\begin{aligned}
& \begin{aligned}
&\left(u_{ \pm}-c\right) \rho_{2 X}+u_{2 X}+v_{2 Y} \\
&=\left(u_{ \pm}-c\right) A_{1 X} \rho_{1 Y}-\left(u_{1} \rho_{1 X}+v_{1} \rho_{1 Y}\right) \\
& \quad-\left(\rho_{1 \bar{t}}+u_{ \pm} \rho_{1 \bar{x}}\right)+A_{1 X} u_{1 Y}-\rho_{1}\left(u_{1 X}+v_{1 Y}\right)-\left(u_{1 \bar{x}}+v_{1 \bar{y}}\right)
\end{aligned} \\
& \left(u_{ \pm}-c\right) u_{2 X}+\frac{1}{\rho_{ \pm} M^{2}} \rho_{2 X} \\
& =\left(u_{ \pm}-c\right) A_{1 X} u_{1 Y}-\left(u_{1} u_{1 X}+v_{1} u_{1 Y}\right) \\
& \quad-\left(u_{1 \bar{t}}+u_{ \pm} u_{1 \bar{x}}\right)+\frac{1}{\rho_{ \pm} M^{2}}\left(-(\gamma-2) \rho_{1} \rho_{1 X}+A_{1 X} \rho_{1 Y}-\rho_{1 \bar{x}}\right)
\end{aligned}
$$

$$
\begin{aligned}
\left(u_{ \pm}-c\right) v_{2 X}+\frac{1}{\rho_{ \pm} M^{2}} \rho_{2 Y} \\
=\left(u_{ \pm}-c\right) A_{1 X} v_{1 Y}-\left(u_{1} v_{1 X}+v_{1} v_{1 Y}\right) \\
\quad-\left(v_{1 \bar{t}}+u_{ \pm} v_{1 \bar{x}}\right)+\frac{1}{\rho_{ \pm} M^{2}}\left(-(\gamma-2) \rho_{1} \rho_{1 Y}-\rho_{1 \bar{y}}\right)
\end{aligned}
$$

Elimination of $u_{2}$ and $v_{2}$ leads to an inhomogeneous wave equation for $\rho_{2}$ :

$$
\begin{aligned}
\beta_{ \pm}^{2} \rho_{2 X X}-\rho_{2 Y Y}= & (\gamma+1) \frac{\rho_{ \pm}^{3}\left(u_{ \pm}-c\right)^{6} M^{6}}{\beta_{ \pm}^{2}}\left(\theta \theta_{\xi}\right)_{\xi} \\
& -\rho_{ \pm}\left(u_{ \pm}-c\right)^{2} M_{ \pm}^{2} \beta_{ \pm}^{2}\left(2 A_{1 X} \theta_{\xi \xi}+A_{1 X X} \theta_{\xi}\right) \\
& -2 \rho_{ \pm}\left(u_{ \pm}-c\right)^{2} M^{2} \theta_{\xi \bar{y}}-\frac{2}{\beta_{ \pm}} \rho_{ \pm}^{2}\left(u_{ \pm}-c\right)^{3} M^{4} \theta_{\xi \bar{t}} \\
& -\frac{2}{\beta_{ \pm}} \rho_{ \pm}\left(u_{ \pm}-c\right)^{2} M^{2}\left(M^{2} \rho_{ \pm} u_{ \pm}\left(u_{ \pm}-c\right)-1\right) \theta_{\xi \bar{x}} .
\end{aligned}
$$


The perturbations are taken to be periodic in $X$ and bounded as $\bar{y} \rightarrow \infty$. In order that the solution for $\rho_{2}$ contain no secular terms that grow indefinitely as $\xi$ increases, the terms on the right-hand side of (3.18) that depend only on $\xi$ (and have no separate dependence on $X$ ) should add to zero:

$$
\begin{gathered}
\frac{(\gamma+1) \rho_{ \pm}^{2}\left(u_{ \pm}-c\right)^{4} M^{4}}{2 \beta_{ \pm}^{2}}\left(\theta \theta_{\xi}\right)_{\xi}-\theta_{\xi \bar{y}}-\frac{1}{\beta_{ \pm}} \rho_{ \pm}\left(u_{ \pm}-c\right) M^{2} \theta_{\xi \bar{z}} \\
-\frac{1}{\beta_{ \pm}}\left(M^{2} \rho_{ \pm} u_{ \pm}\left(u_{ \pm}-c\right)-1\right) \theta_{\xi \bar{x}}=0
\end{gathered}
$$

where $\theta=\left(F_{1 \pm}\right)_{\xi}$, as already noted. This equation describes the slow nonlinear evolution of perturbations in the external flow. With the necessary changes in notation taken into account, it can be shown that the result (3.19) is equivalent to that of [2] in the special case $u_{+}=-u_{-}=T_{ \pm}=1$. The nonlinear term and the term containing a derivative with respect to $\bar{y}$ are the same as for the far field of the flow past a rigid surface; these terms could have been derived instead through matching of an inner solution in terms of $y$ with an outer solution in terms of $\bar{y}$. The remaining terms allow for slow spatial or temporal distortion of the surface. Equation (3.19) can be integrated twice with respect to $\xi$ to give a differential equation for $F_{1}$. The arbitrary function of $\bar{x}$ and $\bar{y}$ arising after one integration is zero if $F_{1}$ is required to be periodic in $\xi$; the second such function remains arbitrary and may be also taken equal to zero, since $F_{1}$ plays the role of a potential function. For consideration of spatial instability, the derivative with respect to $\bar{t}$ is omitted throughout the following.

Equation (3.19), after one integration and with the dependence on $\bar{t}$ omitted, states that $\theta$ is constant along characteristics that can be defined in the form

$$
\begin{aligned}
& \bar{y}=s, \quad \bar{x}=\frac{M^{2} \rho_{ \pm} u_{ \pm}\left(u_{ \pm}-c\right)-1}{\beta_{ \pm}} s+\bar{x}_{0}, \\
& \xi=-\frac{(\gamma+1) \rho_{ \pm}^{2}\left(u_{ \pm}-c\right)^{4} M^{4}}{2 \beta_{ \pm}^{2}} \theta s+X_{0},
\end{aligned}
$$

where $s$ is measured along the characteristics. On a given characteristic, identified by the values of $\bar{x}_{0}$ and $X_{0}, \theta$ is equal to its value at the point where that characteristic intersects the surface $\bar{y}=0$, which in turn equals 
the value of $A_{1 X}$ at that point:

$$
\theta(\xi, \bar{x}, \bar{y})=\theta\left(X_{0}, \bar{x}_{0}, 0\right)=A_{1 X}\left(X_{0}, \bar{x}_{0}\right)
$$

If $A_{1 X}(X, \bar{x})$ were known for one spatial period, say $0<X<1$, and for all $\bar{x}>0$, where the disturbance is regarded as introduced at $\bar{x}=0$, the differential equation (3.19) would allow continuation of the solution away from $\bar{y}=0$. For a vortex sheet, the continuity requirement for $p$ and the kinematic conditions for $v$ would lead to a differential equation for $A_{1}$ equivalent to a result given in [2]. But in the present case the nonlinear external flow is coupled with the flow in a nonlinear critical layer, and so the solutions for the main shear layer and critical layer are needed to provide the initial values of $\theta$ at $\bar{y}=0$ and $\bar{x}>0$.

A particular solution for $\rho_{2}$ is easily found from (3.18), after the terms shown in (3.19) have been omitted; particular solutions for $u_{2}$ and $v_{2}$ then follow from (3.16) and (3.17), and of course are also consistent with (3.15). Solutions to the associated homogeneous equations are chosen to include only outgoing waves, which are represented by undetermined functions of $\xi$ denoted by $F_{2+}$ for $Y>0$ and $F_{2-}$ for $Y<0$; it is anticipated that $F_{2+} \neq F_{2-}$. The full solutions for $\rho_{2}$ and $v_{2}$, with the dependence on $\bar{t}$ omitted, are then

$$
\begin{aligned}
\rho_{2}= & -\rho_{ \pm}\left(u_{ \pm}-c\right)^{2} M^{2} A_{1} \theta_{\xi}-(\gamma-1) \frac{1}{\beta_{ \pm}^{2}} \rho_{ \pm}^{2}\left(u_{ \pm}-c\right)^{4} M^{4} \frac{1}{2} \theta^{2} \\
& +\frac{1}{\beta_{ \pm}} \rho_{ \pm}\left(u_{ \pm}-c\right)^{2} M^{2}\left(F_{2 \pm}\right)_{\xi} \\
v_{2}= & -\beta_{ \pm}\left(u_{ \pm}-c\right) A_{1} \theta_{\xi}-u_{ \pm} F_{1 \bar{X}}-\frac{u_{ \pm}-c}{\beta_{ \pm}} F_{1 \bar{y}}+\left(u_{ \pm}-c\right)\left(F_{2 \pm}\right)_{\xi},
\end{aligned}
$$

where $A_{1}=A_{1}(X, \bar{x})$ and $\theta=F_{1 \xi}=\theta(\xi, \bar{x}, \bar{y})$; the \pm subscripts have now been omitted from $F_{1}$. The particular solution for $\rho_{2}$ is chosen for later convenience to include the second term, as well as the first, in (3.22). The corresponding particular solution for $v_{2}$ then contains the first three terms shown in (3.23). Expressions for $u_{2}$ and $p_{2}$ are also easily found. Related results for second-order supersonic aerodynamics were given by Van Dyke [11].

If, after (3.19) is integrated twice, $F_{1 \bar{y}}$ is eliminated from the solution (3.23) for $v_{2}$, and $p_{2}$ is found by substituting (3.4), (3.11), and (3.22) in 
(2.11), then

$$
\begin{aligned}
v_{2}= & -\beta_{ \pm}\left(u_{ \pm}-c\right) A_{1} \theta_{\xi}+\frac{c}{\beta_{ \pm}^{2}} F_{1 \bar{x}} \\
& -\frac{\gamma+1}{4} \frac{1}{\beta_{ \pm}^{3}} \rho_{ \pm}^{2}\left(u_{ \pm}-c\right)^{5} M^{4} \theta^{2}+\left(u_{ \pm}-c\right)\left(F_{2 \pm}\right)_{\xi} \\
p_{2}= & -\rho_{ \pm}\left(u_{ \pm}-c\right)^{2} \gamma M^{2} A_{1} \theta_{\xi}+\frac{1}{\beta_{ \pm}} \rho_{ \pm}\left(u_{ \pm}-c\right)^{2} \gamma M^{2}\left(F_{2 \pm}\right)_{\xi}
\end{aligned}
$$

The term proportional to $F_{1 \bar{x}}$ agrees, as it should, with the corresponding term given in [7]. The remaining terms can be shown to be consistent with the second-order pressure-angle relation for isentropic flow turning through a simple wave. For points close to the shear layer, i.e., as $Y \rightarrow 0$, the expansions for $v$ and $p$ are found to have the form

$$
\begin{aligned}
& v=\varepsilon\left(u_{ \pm}-c\right)\left[A_{1 X}-\beta_{ \pm} Y A_{1 X X}+\cdots\right] \\
& +\varepsilon^{2}\left[-\left(u_{ \pm}-c\right) \beta_{ \pm} A_{1} A_{1 X X}+\left(u_{ \pm}-c\right)\left(F_{2 \pm}\right)_{X}\right. \\
& \left.\quad+\frac{c}{\beta_{ \pm}^{2}} A_{1 \bar{x}}-\frac{\gamma+1}{4} \frac{1}{\beta_{ \pm}^{3}} \rho_{ \pm}^{2}\left(u_{ \pm}-c\right)^{5} M^{4} A_{1 X}^{2}+\cdots\right]+\cdots \\
& p=1+\varepsilon \gamma M^{2} \frac{1}{\beta_{ \pm}} \rho_{ \pm}\left(u_{ \pm}-c\right)^{2}\left[A_{1 X}-\beta_{ \pm} Y A_{1 X X}+\cdots\right] \\
& +\varepsilon^{2} \gamma M^{2} \frac{1}{\beta_{ \pm}} \rho_{ \pm}\left(u_{ \pm}-c\right)^{2}\left[-\beta_{ \pm} A_{1} A_{1 X X}+\left(F_{2 \pm}\right)_{X}+\cdots\right]
\end{aligned}
$$

where $\left(F_{2 \pm}\right)_{X}$ is now evaluated at $Y=\bar{y}=0$, and $\theta(X, \bar{x}, 0)$ has been replaced by $A_{1 X}(X, \bar{x})$. In contrast to [2], where a vortex sheet is considered, here the shear-layer thickness is not taken to be small in comparison with the displacement of the sheet, and so the expansions (3.26), (3.27) are to be matched with solutions obtained for the main part of the shear layer.

\section{Main shear layer}

Within the shear layer the variables $u, p, \rho$, and $T$ are assumed to possess expansions in terms of the shear-layer variable $\tilde{y}=Y / \delta$ in the form

$$
u=\tilde{u}_{0}(\tilde{y})+\varepsilon \tilde{u}_{1}(X, \tilde{y}, \bar{x})+\cdots
$$




$$
\begin{aligned}
p & =1+\varepsilon \tilde{p}_{1}(X, \tilde{y}, \bar{x})+\varepsilon \delta \tilde{p}_{2}(X, \tilde{y}, \bar{x})+\cdots \\
\rho & =\tilde{\rho}_{0}(\tilde{y})+\varepsilon \tilde{\rho}_{1}(X, \tilde{y}, \bar{x})+\cdots \\
T & =\tilde{T}_{0}(\tilde{y})+\varepsilon \tilde{T}_{1}(X, \tilde{y}, \bar{x})+\cdots
\end{aligned}
$$

where again the perturbations depend on the parameter $\varepsilon / \delta=O(1)$ as well as on the variables shown. The formulation resembles closely that of [7], which also considers a long-wave limit, but certain differences appear because the displacement of the shear layer is now larger than in [7], and a displacement term $y=A$ has therefore been subtracted in the definition (2.3) for $Y$. It is convenient to define a transformed velocity $V$ by subtracting certain terms to allow cancellation of terms introduced in the differential equations as a result of the coordinate shift $Y=y-A$. Thus we also set

$$
v-(u-c) A_{X}-\varepsilon u A_{\bar{x}}=V=\varepsilon \delta \tilde{V}_{2}(X, \tilde{y}, \bar{x})+\cdots,
$$

where the continuity equation has been used to show that the expansion of $V$ contains no term of order $\varepsilon$; this is consistent with the anticipated result (3.13). As in the case of the external flow, the orders of subsequent terms are in fact not determined until the solutions for earlier terms have been completed. In particular, each of the functions $\tilde{u}_{1}, \tilde{p}_{2}, \tilde{\rho}_{1}, \tilde{T}_{1}$, and $\tilde{V}_{2}$ is found to contain a term proportional to $\ln \varepsilon$; as in Section 3, these terms are not shown explicitly.

It is easily seen from the transverse momentum equation that $\tilde{p}_{1}$ is constant across the shear layer. Matching with the external-flow solution (3.27) for the pressure then shows that the quantity $\rho_{ \pm}\left(u_{ \pm}-c\right)^{2} / \beta_{ \pm}$has the same value, say $\Gamma$, both above and below the shear layer, and that

$$
\tilde{p}_{1}=\gamma M^{2} \Gamma A_{1 X}
$$

where $\Gamma$ is defined by

$$
\Gamma=\frac{\rho_{+}\left(u_{+}-c\right)^{2}}{\beta_{+}}=\frac{\rho_{-}\left(u_{-}-c\right)^{2}}{\beta_{-}} .
$$

Combining (4.7) with the definition (3.10) of $\beta_{ \pm}$gives $\left(\beta_{+}-\beta_{-}\right)(1-$ $\left.\beta_{+} \beta_{-}\right)=0$; the case $\beta_{+} \neq \beta_{-}$leads to a quartic equation for $c$,

$$
\frac{1}{M^{2} \rho_{+}\left(u_{+}-c\right)^{2}}+\frac{1}{M^{2} \rho_{-}\left(u_{-}-c\right)^{2}}=1,
$$

in agreement with the results cited earlier for waves on a vortex sheet [1] 
and for long-wave disturbances on a shear layer [5]. As anticipated in (3.9), each term on the left side of (4.8) is smaller than one. Explicit solutions for $c$ can be found in special cases [1].

The next terms in the expansions of the continuity and $\tilde{y}$-momentum equations and the first approximations to the $X$-momentum and energy equations are

$$
\begin{array}{r}
\tilde{u}_{1 X}+\tilde{V}_{2 \bar{y}}+\frac{1}{\gamma}\left(\tilde{u}_{0}-c\right) \tilde{p}_{1 X}=0 \\
\left(\tilde{u}_{0}-c\right)^{2} A_{1 X X}+\frac{1}{\gamma M^{2}} \frac{1}{\tilde{\rho}_{0}} \tilde{p}_{2 \bar{y}}=0 \\
\left(\tilde{u}_{0}-c\right) \tilde{u}_{1 X}+\tilde{V}_{2} \tilde{u}_{0}^{\prime}+\frac{1}{\gamma M^{2}} \frac{1}{\tilde{\rho}_{0}} \tilde{p}_{1 X}=0 \\
\left(\tilde{u}_{0}-c\right) \tilde{T}_{1 X}+\tilde{V}_{2} \tilde{T}_{0}^{\prime}-\frac{\gamma-1}{\gamma}\left(\tilde{u}_{0}-c\right) \frac{1}{\tilde{\rho}_{0}} \tilde{p}_{1 X}=0
\end{array}
$$

where $\tilde{u}_{0}^{\prime}$ and $\tilde{T}_{0}^{\prime}$ denote derivatives with respect to $\tilde{y}$. The leading terms in the expansion of the equation of state are

$$
\tilde{\rho}_{0} \tilde{T}_{0}=1, \quad \tilde{p}_{1}=\frac{\tilde{\rho}_{1}}{\tilde{\rho}_{0}}+\frac{\tilde{T}_{1}}{\tilde{T}_{0}} .
$$

These equations, along with the energy equation (4.12), have been used to eliminate $\tilde{\rho}_{1}$ from the continuity equation (4.9).

Elimination of $\tilde{u}_{1}$ from the continuity and $X$-momentum equations (4.9) and (4.11) now leads to an equation for $\tilde{V}_{2}$ that can be integrated to give

$$
\begin{aligned}
\frac{\tilde{V}_{2}}{\tilde{u}_{0}-c}= & \frac{\tilde{p}_{1 X}}{\gamma M^{2} \rho_{ \pm}\left(u_{ \pm}-c\right)^{2}}\left(-\beta_{ \pm}^{2} \tilde{y}+\int_{ \pm \infty}^{\tilde{y}}\left(\frac{\rho_{ \pm}\left(u_{ \pm}-c\right)^{2}}{\tilde{\rho}_{0}\left(\tilde{u}_{0}-c\right)^{2}}-1\right) d \tilde{y}\right) \\
& +\left(G_{2 \pm}\right)_{X},
\end{aligned}
$$

where $G_{2 \pm}=G_{2 \pm}(X, \bar{x})$ and is to be determined; the upper and lower signs are to be taken, respectively, for $\tilde{y}>0$ and $\tilde{y}<0$. It is seen in Section 5 that each of the functions $G_{2 \pm}$ includes terms containing a factor ln $\varepsilon$. The results for $\tilde{p}_{2}, \tilde{u}_{1}$, and $\tilde{T}_{1}$ are found, from (4.10), (4.11), and (4.12), respectively, to be

$$
\tilde{p}_{2}=P_{2 \pm}-\gamma M^{2} \rho_{ \pm}\left(u_{ \pm}-c\right)^{2} A_{1 X X}\left(\tilde{y}+\int_{ \pm \infty}^{\tilde{y}}\left(\frac{\tilde{\rho}_{0}\left(\tilde{u}_{0}-c\right)^{2}}{\rho_{ \pm}\left(u_{ \pm}-c\right)^{2}}-1\right) d \tilde{y}\right)
$$




$$
\begin{aligned}
\tilde{u}_{1}= & -\frac{\tilde{p}_{1} \tilde{u}_{0}^{\prime}}{\gamma M^{2} \rho_{ \pm}\left(u_{ \pm}-c\right)^{2}}\left(-\beta_{ \pm}^{2} \bar{y}+\int_{ \pm \infty}^{\tilde{y}}\left(\frac{\rho_{ \pm}\left(u_{ \pm}-c\right)^{2}}{\tilde{\rho}_{0}\left(\tilde{u}_{0}-c\right)^{2}}-1\right) d \tilde{y}\right) \\
& -\frac{\tilde{p}_{1}}{\gamma M^{2} \tilde{\rho}_{0}\left(\tilde{u}_{0}-c\right)}-G_{2 \pm} \tilde{u}_{0}^{\prime} \\
\tilde{T}_{1}= & -\frac{\tilde{p}_{1} \tilde{T}_{0}^{\prime}}{\gamma M^{2} \rho_{ \pm}\left(u_{ \pm}-c\right)^{2}}\left(-\beta_{ \pm}^{2} \tilde{y}+\int_{ \pm \infty}^{\tilde{y}}\left(\frac{\rho_{ \pm}\left(u_{ \pm}-c\right)^{2}}{\tilde{\rho}_{0}\left(\tilde{u}_{0}-c\right)^{2}}-1\right) d \tilde{y}\right) \\
& +\frac{\gamma-1}{\gamma} \frac{\tilde{p}_{1}}{\tilde{\rho}_{0}}-G_{2 \pm} \tilde{T}_{0}^{\prime}
\end{aligned}
$$

where $P_{2 \pm}=P_{2 \pm}(X, \bar{x})$ and is to be determined. The integrals shown here are equivalent to those arising in the long-wave derivation of [5]. All the terms in (4.14) through (4.17) appear also in the expressions given by Balsa [7]; although the limiting cases considered are different, these aspects of the disturbances to the main shear layer are the same. Additional terms that appear in [7] are absent here as a result of the coordinate change from $y / \delta$ to $Y / \delta$.

Since $\tilde{u}_{0}^{\prime} \rightarrow 0$ and $\tilde{T}_{0}^{\prime} \rightarrow 0$ as $\tilde{y} \rightarrow \pm \infty$, it is easy to determine the behavior of $\tilde{u}_{1}$ and $\tilde{T}_{1}$ for large $\tilde{y}$, and then to verify that the solutions for $u$ and $T$ match properly with solutions obtained for the external flow. But matching of the solutions for $v$ and $p$ as $Y \rightarrow 0$ and $\tilde{y} \rightarrow \pm \infty$ is possible only if certain relationships among the unknown functions are satisfied. With the help of (4.5) and (4.6), the shear-layer expansions as $\tilde{y} \rightarrow \pm \infty$ are found to have the form

$$
\begin{aligned}
& v= \varepsilon\left(u_{ \pm}-c\right) A_{1 X}+\varepsilon^{2}\left(\left(u_{ \pm}-c\right) A_{2 X}-\frac{u_{ \pm}-c}{\beta_{ \pm}} A_{1 X}^{2}+u_{ \pm} A_{1 \tilde{x}}\right)+\cdots \\
&+\varepsilon \delta\left(u_{ \pm}-c\right)\left(A_{1 X X}\left(-\beta_{ \pm} \tilde{y}\right)+\left(G_{2 \pm}\right)_{X}\right)+\cdots \\
& p=1+\varepsilon \gamma M^{2} \rho_{ \pm}\left(u_{ \pm}-c\right)^{2} \frac{1}{\beta_{ \pm}} A_{1 X} \\
& \quad+\varepsilon \delta\left(-\gamma M^{2} \rho_{ \pm}\left(u_{ \pm}-c\right)^{2} A_{1 X X} \tilde{y}+P_{2 \pm}\right)+\cdots
\end{aligned}
$$

From comparison of (3.26) and (3.27) for the external flow with (4.18) and (4.19) for the shear layer, expressions for $(\delta / \varepsilon) G_{2 \pm}(X, \bar{x}, 0)+A_{2}(X, \bar{x})$ and 
$P_{2 \pm}(X, \bar{x})$ are found in terms of $F_{2 \pm}(X, \bar{x}, 0)$ and $A_{1}(X, \bar{x})$ :

$$
\begin{aligned}
\frac{\delta}{\varepsilon}\left(G_{2_{ \pm}}\right)_{X}+A_{2 X}= & C_{ \pm} A_{1 \bar{x}}+\left(F_{2 \pm}\right)_{X}-\beta_{ \pm} A_{1} A_{1 X X} \\
& +\frac{1}{\beta_{ \pm}}\left(1-\frac{\gamma+1}{4} \Gamma^{2} M^{4}\right) A_{1 X}^{2} \\
P_{2 \pm}= & \gamma M^{2} \frac{\varepsilon}{\delta} \Gamma\left(\left(F_{2 \pm}\right)_{X}-\beta_{ \pm} A_{1} A_{1 X X}\right)
\end{aligned}
$$

where the constants $C_{ \pm}$are defined by

$$
C_{ \pm}=\frac{1}{u_{ \pm}-c}\left(\frac{c}{\beta_{ \pm}^{2}}-u_{ \pm}\right)=-1+\frac{c}{u_{ \pm}-c}\left(\beta_{\mp}^{2}-1\right)
$$

and the result $\beta_{+} \beta_{-}=1$ has been used. Each of the quantities $\varepsilon \delta\left(G_{2 \pm}\right)_{X}$ and $\varepsilon^{2} A_{2 X}$ represents a contribution to the streamline slope in the flow at the edge of the shear layer, and it is seen that the two terms appear together in (4.20), suggesting an indeterminacy. As noted again in Section 5, the definition of $A$ can be made precise if $y=A$ (i.e., $Y=0$ ) is identified as the line where $u-c$ is exactly zero; then $\varepsilon \delta\left(G_{2 \pm}\right)$ represents a term in streamline displacement relative to the line $y=A$. The jump $G_{2+}-G_{2-}$, rather than the functions $G_{2+}$ and $G_{2-}$ separately, will be needed in the derivation of an evolution equation for $A_{1}$ in Section 5. Combining (4.20) and (4.21), and using the fact that $\beta_{+} \beta_{-}=1$ in the final term,

$$
\begin{aligned}
\frac{\delta}{\varepsilon}\left(G_{2+}-G_{2-}\right)_{X}= & \left(C_{+}-C_{-}\right) A_{1 \bar{x}}+\frac{\delta}{\varepsilon} \frac{1}{\Gamma} \frac{P_{2+}-P_{2-}}{\gamma M^{2}} \\
& -\left(\beta_{+}-\beta_{-}\right)\left(1-\frac{\gamma+1}{4} \Gamma^{2} M^{4}\right) A_{1 X}^{2}
\end{aligned}
$$

The behavior of the shear-layer solutions for small $\tilde{y}$ is also needed. As $\tilde{y} \rightarrow 0$, expansion of $\tilde{u}_{0}$ and $\tilde{T}_{0}$ in Taylor series shows that the integral appearing in $\tilde{V}_{2}, \tilde{u}_{1}$, and $\tilde{T}_{1}$ is $O(1 / \tilde{y})$. One way of removing the singular part is shown in [7]; a somewhat different procedure is used here, leading to expansions of the perturbation quantities as $\tilde{y} \rightarrow 0$ in the form

$$
\begin{aligned}
& \tilde{V}_{2}=-\frac{1}{\tilde{\rho}_{0 \mathrm{c}} \tilde{u}_{0 \mathrm{c}}^{\prime}} \Gamma A_{1 X X}\left(1+\left(\frac{\tilde{u}_{0 \mathrm{c}}^{\prime \prime}}{\tilde{u}_{0 \mathrm{c}}^{\prime}}-\frac{\tilde{T}_{0 \mathrm{c}}^{\prime}}{\tilde{T}_{0 \mathrm{c}}}\right) \tilde{y} \ln |\tilde{y}|\right)+O(\tilde{y}) \\
& \tilde{p}_{2}=P_{2 \pm}-\gamma M^{2} \Gamma A_{1 X X} K_{ \pm}+o(1)
\end{aligned}
$$




$$
\begin{aligned}
\tilde{u}_{1}= & \frac{1}{\tilde{\rho}_{0 \mathrm{c}} \tilde{u}_{0 \mathrm{c}}^{\prime}} \Gamma A_{1 X}\left(\left(\frac{\tilde{u}_{0 \mathrm{c}}^{\prime \prime}}{\tilde{u}_{0 \mathrm{c}}^{\prime}}-\frac{\tilde{T}_{0 \mathrm{c}}^{\prime}}{\tilde{T}_{0 \mathrm{c}}}\right) \ln |\tilde{y}|+\frac{3 \tilde{u}_{0 \mathrm{c}}^{\prime \prime}}{2 \tilde{u}_{0 \mathrm{c}}^{\prime}}-\frac{\tilde{T}_{0 \mathrm{c}}^{\prime}}{\tilde{T}_{0 \mathrm{c}}}\right) \\
& -A_{1 X} \tilde{u}_{0 \mathrm{c}}^{\prime} J_{ \pm}-G_{2 \pm} \tilde{u}_{0 \mathrm{c}}^{\prime}+o(1) \\
\tilde{T}_{1}= & \tilde{T}_{0 \mathrm{c}}^{\prime} \frac{1}{\tilde{\rho}_{0 \mathrm{c}} \tilde{u}_{0 \mathrm{c}}^{\prime 2}} \Gamma A_{1 X}\left(\frac{1}{\tilde{y}}+\left(\frac{\tilde{u}_{0 \mathrm{c}}^{\prime \prime}}{\tilde{u}_{0 \mathrm{c}}^{\prime}}-\frac{\tilde{T}_{0 \mathrm{c}}^{\prime}}{\tilde{T}_{0 \mathrm{c}}}\right) \ln |\tilde{y}|+O(1)\right),
\end{aligned}
$$

where only those terms that may be needed later have been shown. The subscript $\mathrm{c}$ indicates that a function of $\tilde{y}$ is to be evaluated at the location of the critical layer, namely at $\tilde{y}=0$, and the integrals $J_{ \pm}$and $K_{ \pm}$in $\tilde{u}_{1}$ and $\tilde{p}_{2}$ are defined by

$$
\begin{aligned}
& J_{ \pm}=\frac{1}{\beta_{ \pm}} \int_{ \pm \infty}^{0}\left\{\frac{\rho_{ \pm}\left(u_{ \pm}-c\right)^{2}}{\tilde{\rho}_{0}\left(\tilde{u}_{0}-c\right)^{2}}-1\right. \\
& \quad-\frac{\rho_{ \pm}}{\tilde{\rho}_{0 \mathrm{c}} \tilde{u}_{0 \mathrm{c}}^{\prime 2}}\left(\frac{\left(u_{ \pm}-c\right)^{2}-\left(\tilde{u}_{0}-c\right)^{2}}{\tilde{y}^{2}}+\frac{2\left(\tilde{u}_{0}-c\right) \tilde{u}_{0}^{\prime}}{\tilde{y}}\right) \\
&\left.+\frac{\rho_{ \pm}\left(u_{ \pm}-c\right)}{\tilde{\rho}_{0 \mathrm{c}} \tilde{u}_{0 \mathrm{c}}^{\prime 2}}\left(\frac{\tilde{u}_{0 \mathrm{c}}^{\prime \prime}}{\tilde{u}_{0 c}^{\prime}}-\frac{\tilde{T}_{0 \mathrm{c}}^{\prime}}{\tilde{T}_{0 \mathrm{c}}}\right)\left(\frac{u_{ \pm}-\tilde{u}_{0}}{\tilde{y}}-\tilde{u}_{0}^{\prime} \ln |\tilde{y}|\right)\right\} d \tilde{y} \\
& K_{ \pm}=\beta_{ \pm} \int_{ \pm \infty}^{0}\left(\frac{\tilde{\rho}_{0}\left(\tilde{u}_{0}-c\right)^{2}}{\rho_{ \pm}\left(u_{ \pm}-c\right)^{2}}-1\right) d \tilde{y} .
\end{aligned}
$$

The integrands in $J_{ \pm}$have been modified so as to be integrable at $\tilde{y}=0$ and also to approach zero exponentially as $\tilde{y} \rightarrow \infty$ (if $\tilde{u}_{0}-u_{ \pm} \rightarrow 0$ and $\tilde{T}_{0}-T_{ \pm} \rightarrow$ 0 exponentially); the first few terms in $\tilde{u}_{1}$ appear because of these changes. The vorticity $(2.12)$ as $\tilde{y} \rightarrow 0$ is

$$
\Omega=-\frac{1}{\delta}\left(\tilde{u}_{0 \mathrm{c}}^{\prime}+\tilde{u}_{0 \mathrm{c}}^{\prime \prime} \tilde{y}+\cdots\right)-\frac{\varepsilon}{\delta} \frac{1}{\tilde{\rho}_{0 \mathrm{c}} \tilde{u}_{0 \mathrm{c}}^{\prime}} \Gamma A_{1 X}\left(\frac{\tilde{u}_{0 \mathrm{c}}^{\prime \prime}}{\tilde{u}_{0 \mathrm{c}}^{\prime}}-\frac{\tilde{T}_{0 \mathrm{c}}^{\prime}}{\tilde{T}_{0 \mathrm{c}}}\right) \frac{1}{\tilde{y}}+\cdots
$$

An approximate stream function is useful in Section 5 for obtaining solutions of the energy and vorticity equations in the critical layer. In terms of variables $X, Y$, and $\bar{x}$, the continuity equation is

$$
[\rho(u-c)]_{X}+(\rho V)_{Y}+\varepsilon(\rho u)_{\bar{x}}=0
$$


For $\tilde{y}=O(1)$ the solutions derived above show that the third term in (4.31) is $O\left(\varepsilon^{3 / 2}\right)$, i.e., of higher order than the first two terms. Thus each of the two mass-flow components $\rho(u-c)$ and $\rho V$ can be written in terms of a stream function $\psi$, or $\tilde{\psi}=\psi / \delta$ if the appropriate scaling for the shear layer is introduced, plus a higher-order correction term:

$$
\begin{aligned}
\rho(u-c) & =\psi_{Y}+\cdots=\tilde{\psi}_{\tilde{y}}+\cdots \\
\rho V & =-\psi_{X}+\cdots=-\delta \tilde{\psi}_{X}+\cdots .
\end{aligned}
$$

Substitution of the expansions for $\rho, u$, and $V$ into (4.32) allows calculation of derivatives of $\tilde{\psi}$ as $\tilde{y} \rightarrow 0$ :

$$
\tilde{\psi}_{\tilde{y}}=\tilde{\rho}_{0 \mathrm{c}} \tilde{u}_{0 \mathrm{c}}^{\prime} \tilde{y}+\cdots, \quad \tilde{\psi}_{X}=\varepsilon \frac{1}{\tilde{u}_{0 \mathrm{c}}^{\prime}} \Gamma A_{1 X X}+\cdots
$$

Thus, as $\tilde{y} \rightarrow 0$,

$$
\tilde{\psi}=\frac{1}{2} \tilde{\rho}_{0 \mathrm{c}} \tilde{u}_{0 \mathrm{c}}^{\prime} \tilde{y}^{2}+O\left(\tilde{y}^{3}, \varepsilon\right)
$$

\section{Critical layer}

\subsection{Temperature and vorticity}

One way to recognize the scale for the critical layer is to note from (4.34) that the first approximation for $\tilde{\psi}$ is no longer given by the undisturbed value when $\tilde{y}=O\left(\varepsilon^{1 / 2}\right)$ and therefore $\tilde{\psi}=O(\varepsilon)$. This is the expected scale for the thickness of a nonlinear critical layer, as given first by Benney and Bergeron [12]. Thus an appropriate coordinate for the critical layer is $\hat{y}=\tilde{y} / \varepsilon^{1 / 2}$, as anticipated in (2.5). The orders of the largest flow perturbations in the critical layer are determined by matching with the solutions for the main part of the shear layer. The asymptotic expansions are then assumed to have the form

$$
\begin{aligned}
u & =c+\varepsilon^{1 / 2} \tilde{u}_{0 c}^{\prime} \hat{y}+\varepsilon \hat{u}_{1}(X, \hat{y}, \bar{x})+\cdots \\
p & =1+\varepsilon \hat{p}_{1}(X, \hat{y}, \bar{x})+\varepsilon \delta \hat{p}_{2}(X, \hat{y}, \bar{x})+\cdots \\
V & =\varepsilon \delta \hat{V}_{2}(X, \hat{y}, \bar{x})+\varepsilon^{3 / 2} \delta \hat{V}_{5 / 2}(X, \hat{y}, \bar{x})+\cdots \\
T & =\tilde{T}_{0 \mathrm{c}}+\varepsilon^{1 / 2} \hat{T}_{1 / 2}(X, \hat{y}, \bar{x})+\varepsilon \hat{T}_{1}(X, \hat{y}, \bar{x})+\cdots
\end{aligned}
$$




$$
\begin{aligned}
\rho & =\tilde{\rho}_{0 c}+\varepsilon^{1 / 2} \hat{\rho}_{1 / 2}(X, \hat{y}, \bar{x})+\varepsilon \hat{\rho}_{1}(X, \hat{y}, \bar{x})+\cdots \\
\Omega & =-\frac{1}{\delta} \tilde{u}_{0 c}^{\prime}+\frac{\varepsilon^{1 / 2}}{\delta} \hat{\Omega}_{1 / 2}(X, \hat{y}, \bar{x})+\frac{\varepsilon}{\delta} \hat{\Omega}_{1}(X, \hat{y}, \bar{x})+\cdots \\
\frac{\tilde{\psi}}{\varepsilon} & =\hat{\psi}=\hat{\psi}_{0}(X, \hat{y}, \bar{x})+\varepsilon^{1 / 2} \hat{\psi}_{1 / 2}(X, \hat{y}, \bar{x})+\cdots
\end{aligned}
$$

The largest terms in the temperature and the vorticity have been taken as constants, equal to the values in the undisturbed flow close to $\tilde{y}=0$; from the definition (2.12) of the vorticity, the largest term in the velocity difference $u-c$ is then linear in $\hat{y}$. In the expansion of $p$, it has been anticipated that there will be no term of order $\varepsilon^{3 / 2}$, since terms of this order are not needed in $p$ for the external flow or the main part of the shear layer. Certain functions appearing in the asymptotic expansions for these other parts of the flow will be found to contain a factor $\ln \varepsilon$, since, as seen below, the matching of solutions for the critical layer and the main part of the shear layer requires the appearance of a term proportional to $\ln \varepsilon$ in each of the functions $G_{2 \pm}$. The factor $\ln \varepsilon$ is not, however, present in any of the functions shown in the expansions (5.1) through (5.7) above.

The first two terms in the $y$-momentum equation (2.8) show that $\hat{p}_{1}$ and $\hat{p}_{2}$ are independent of $\hat{y}$, i.e., are constant across the critical layer. Matching as $\hat{y} \rightarrow \pm \infty$ and $\tilde{y} \rightarrow 0$ then shows that $\hat{p}_{1}$ is equal to $\tilde{p}_{1}$ and that $\hat{p}_{2}$ is equal to the value of $\tilde{p}_{2}$ at $\tilde{y}=0$; the latter also of course implies that there is no jump in $\tilde{p}_{2}$ across the critical layer. From (4.6) and (4.25), it follows that

$$
\begin{aligned}
\hat{p}_{1} & =\gamma M^{2} \Gamma A_{1 X} \\
P_{2+}-P_{2-} & =\gamma M^{2} \Gamma A_{1 X X}\left(K_{+}-K_{-}\right) .
\end{aligned}
$$

Substitution of (5.9) into (4.23) leads to an expression for the jump $G_{2+}-$ $G_{2-}$ in terms of derivatives of $A_{1}$ and known quantities. The largest term in the continuity equation (2.6) shows that also $\hat{V}_{2}$ is independent of $\hat{y}$, i.e., is constant across the critical layer. Matching as $\hat{y} \rightarrow \pm \infty$ and $\tilde{y} \rightarrow 0$, with the help of (4.24), shows that

$$
\hat{V}_{2}=-\frac{1}{\tilde{\rho}_{0 \mathrm{c}} \tilde{u}_{0 \mathrm{c}}^{\prime}} \Gamma A_{1 X X}
$$

The definition (4.32) of the stream function can be rewritten in terms of $\hat{\psi}$, where, as before, the stream function satisfies the continuity equation only approximately, because the higher-order term $\varepsilon(\rho u)_{\bar{x}}$ in (4.31) has not been taken into account. The largest terms give

$$
\hat{\psi}_{0 \hat{y}}=\tilde{\rho}_{0 \mathrm{c}} \tilde{\boldsymbol{u}}_{0 \mathrm{c}}^{\prime} \hat{y}, \quad \hat{\psi}_{0 X}=-\tilde{\rho}_{0 \mathrm{c}} \hat{V}_{2}
$$


Thus in the first approximation the streamlines are lines with slopes $d \hat{y} / d X=\hat{V}_{2} /\left(\tilde{u}_{0 c}^{\prime} \hat{y}\right)$. The first approximation to the stream function is then

$$
\hat{\psi}_{0}=\frac{1}{2} \tilde{\rho}_{0 \mathrm{c}} \tilde{u}_{0 \mathrm{c}}^{\prime} \hat{y}^{2}+\hat{\psi}_{00}, \quad \hat{\psi}_{00}=\frac{1}{\tilde{u}_{0 \mathrm{c}}^{\prime}} \Gamma\left(A_{1 X}-\left(A_{1 X}\right)_{\mathrm{m}}\right),
$$

where, for each $X, \hat{\psi}_{00}$ is the value of $\hat{\psi}_{0}$ at $\hat{y}=0$, and $\left(A_{1 X}\right)_{m}$ is an integration constant (which may be a function of $\bar{x}$ ) defined as equal to the maximum value of $A_{1 X}$ in one period, say for $0 \leq X \leq 1$; this choice is made so that $\hat{\psi}_{00} \leq 0$. Also, it is seen that the derivatives of (5.12) match properly with (4.33) as $\hat{y} \rightarrow \pm \infty$ and $\tilde{y} \rightarrow 0$. Since $A_{1}$ is periodic in $X$, the streamlines have a cat's-eye structure. The saddle points and centers of the cat's eyes are the points where $\hat{\psi}_{00}, \hat{p}_{1}$, and $A_{1 X}$ reach their maximum or minimum values respectively; $\hat{\psi}_{00}=0$ at the saddle points and $\hat{\psi}_{00}<0$ elsewhere. For each $X$, the approximate stream function $\hat{\psi}_{0}$ lies in the range $\hat{\psi}_{00} \leq \hat{\psi}_{0}<\infty$, with $\hat{\psi}_{0}=0$ at the boundaries of the cat's eyes.

The first approximations to the energy and vorticity equations (2.9) and (2.13) are given by

$$
\begin{array}{r}
\tilde{u}_{0 \mathrm{c}}^{\prime} \hat{y}\left(\hat{T}_{1 / 2}\right)_{X}+\hat{V}_{2}\left(\hat{T}_{1 / 2}\right)_{\hat{y}}=0 \\
\tilde{u}_{0 \mathrm{c}}^{\prime} \hat{y}\left(\hat{\Omega}_{1 / 2}\right)_{X}+\hat{V}_{2}\left(\hat{\Omega}_{1 / 2}\right)_{\hat{y}}-\frac{1}{\gamma M^{2}} \hat{p}_{1 X}\left(\hat{T}_{1 / 2}\right)_{\hat{y}}=0,
\end{array}
$$

where, from (5.8) and (5.10), $\hat{p}_{1 X}$ and $\hat{V}_{2}$ are proportional to $A_{1 X X}$; the equation of state (2.10) and the result (5.13) have been used in obtaining (5.14). Thus the temperature perturbation is constant along the approximate streamlines, and the vorticity changes along a streamline as a result of the baroclinic torque. That is, $\hat{T}_{1 / 2}$ and the unforced term in $\hat{\Omega}_{1 / 2}$ are functions of $\hat{\psi}_{0}$ and $\bar{x}$, and the solutions have the form

$$
\begin{aligned}
& \frac{\hat{T}_{1 / 2}}{\tilde{T}_{0 \mathrm{c}}}=\hat{\Theta}_{1 / 2 \pm}\left(\hat{\psi}_{0}, \bar{x}\right)=-\frac{\hat{\rho}_{1 / 2}}{\tilde{\rho}_{0 \mathrm{c}}} \\
& \hat{\Omega}_{1 / 2}=\frac{1}{\gamma M^{2}} \hat{p}_{1}\left(\hat{\Theta}_{1 / 2 \pm}\right)_{\hat{\psi}_{0}}+\hat{Q}_{1 / 2 \pm}\left(\hat{\psi}_{0}, \bar{x}\right),
\end{aligned}
$$

where the particular solution for $\hat{\Omega}_{1 / 2}$ can be verified by direct substitution, with the help of (5.12); the equation of state (2.10) has been used to relate $\hat{\rho}_{1 / 2}$ and $\hat{T}_{1 / 2}$, and the functions $\hat{\Theta}_{1 / 2 \pm}\left(\hat{\psi}_{0}, \bar{x}\right)$ and $\hat{Q}_{1 / 2 \pm}\left(\hat{\psi}_{0}, \bar{x}\right)$ are still to be determined. The notation $\hat{\Theta}_{1 / 2 \pm}, \hat{Q}_{1 / 2 \pm}$ is intended to emphasize that 
these are different functions of $\hat{\psi}_{0}$ above and below the cat's eyes; in each of these regions $0<\hat{\psi}_{0}<\infty$, while within the cat's eyes $\hat{\psi}_{00} \leq \hat{\psi}_{0}<0$.

The second-order energy and vorticity equations are

$$
\begin{aligned}
& \tilde{u}_{0 \mathrm{c}}^{\prime} \hat{y} \hat{T}_{1 X}+\hat{V}_{2} \hat{T}_{1 \hat{y}}=-\hat{u}_{1}\left(\hat{T}_{1 / 2}\right)_{X}-\hat{V}_{5 / 2}\left(\hat{T}_{1 / 2}\right)_{\hat{y}}-c\left(\hat{T}_{1 / 2}\right)_{\bar{x}} \\
&+\frac{\gamma-1}{\gamma} \tilde{u}_{0 \mathrm{c}}^{\prime} \hat{y} \frac{1}{\tilde{\rho}_{0 \mathrm{c}}} \hat{p}_{1 X}+\frac{1}{\varepsilon^{2} \delta^{2} \operatorname{Re} \operatorname{Pr}} \frac{\tilde{\mu}_{0 \mathrm{c}}}{\tilde{\rho}_{0 \mathrm{c}}}\left(\hat{T}_{1 / 2}\right)_{\hat{y} \bar{y}} \\
& \tilde{u}_{0 \mathrm{c}}^{\prime} \hat{y}\left(\hat{\Omega}_{1}+\tilde{u}_{0 \mathrm{c}}^{\prime} \frac{\hat{\rho}_{1}}{\tilde{\rho}_{0 \mathrm{c}}}\right)_{X}+\hat{V}_{2}\left(\hat{\Omega}_{1}+\tilde{u}_{0 \mathrm{c}}^{\prime} \frac{\hat{\rho}_{1}}{\tilde{\rho}_{0 \mathrm{c}}}\right)_{\hat{y}}-\frac{1}{\gamma M^{2}} \hat{p}_{1 X} \hat{T}_{1 \hat{y}} \\
&=-\hat{u}_{1}\left(\hat{\Omega}_{1 / 2}-\tilde{u}_{0 \mathrm{c}}^{\prime} \frac{\hat{T}_{1 / 2}}{\tilde{T}_{0 \mathrm{c}}}\right)_{X}-\hat{V}_{5 / 2}\left(\hat{\Omega}_{1 / 2}-\tilde{u}_{0 \mathrm{c}}^{\prime} \frac{\hat{T}_{1 / 2}}{\tilde{T}_{0 \mathrm{c}}}\right)_{\hat{y}} \\
&-c\left(\hat{\Omega}_{1 / 2}-\tilde{u}_{0 \mathrm{c}}^{\prime} \frac{\hat{T}_{1 / 2}}{\tilde{T}_{0 \mathrm{c}}}\right)_{\bar{x}}+\frac{1}{\varepsilon^{2} \delta^{2} \operatorname{Re}} \frac{\tilde{\mu}_{0 \mathrm{c}}}{\tilde{\rho}_{0 \mathrm{c}}}\left(\hat{\Omega}_{1 / 2}\right)_{\hat{y} \hat{y}}
\end{aligned}
$$

where $\hat{T}_{1 / 2}$ and $\hat{\Omega}_{1 / 2}$ are given in terms of $\hat{\Theta}_{1 / 2 \pm}$ and $\hat{Q}_{1 / 2 \pm}$ by $(5.15)$ and (5.16); Eqs. (5.13) and (5.15) have been used in simplifying (5.18), and $\hat{\rho}_{1}$ is related to $\hat{p}_{1}$ and $\hat{T}_{1}$ through the equation of state (2.10). Since $\hat{\Theta}_{1 / 2 \pm}$ and $\hat{Q}_{1 / 2 \pm}$ are functions of $\hat{\psi}_{0}$, derivatives of these quantities with respect to $\hat{y}$ are found using $\partial / \partial \hat{y}=\tilde{\rho}_{0 c} \tilde{u}_{0 c}^{\prime} \hat{y} \partial / \partial \hat{\psi}_{0}$.

In each of (5.17) and (5.18) the first two terms on the right-hand side can be rewritten and grouped with the terms on the left-hand side. Combining the second terms in the definition of the stream function $\hat{\psi}$ with (5.11) gives

$$
\hat{u}_{1} \hat{\psi}_{0 X}+\hat{V}_{5 / 2} \hat{\psi}_{0 \hat{y}}=-\tilde{u}_{0 \mathrm{c}}^{\prime} \hat{y}\left(\hat{\psi}_{1 / 2}\right)_{X}-\hat{V}_{2}\left(\hat{\psi}_{1 / 2}\right)_{\hat{y}}
$$

(This is simply the second approximation to the statement that the stream function is constant along a streamline.) Equations (5.17) and (5.18) then can be shown to become

$$
\begin{aligned}
& \left.\left(\hat{T}_{1}-\frac{\hat{\psi}_{1 / 2}}{\tilde{\rho}_{0 \mathrm{c}} \tilde{u}_{0 \mathrm{c}}^{\prime} \hat{y}}\left(\hat{T}_{1 / 2}\right)_{\hat{y}}-\frac{\gamma-1}{\gamma} \frac{1}{\tilde{\rho}_{0 \mathrm{c}}} \hat{p}_{1}\right)_{X}\right|_{\hat{\psi}_{0}} \\
& =-\frac{c}{\tilde{u}_{0 \mathrm{c}}^{\prime} \hat{y}}\left(\hat{T}_{1 / 2}\right)_{\bar{x}}+\frac{1}{\varepsilon^{2} \delta^{2} \operatorname{Re} \operatorname{Pr}} \frac{\tilde{\mu}_{0 \mathrm{c}}}{\tilde{\rho}_{0 \mathrm{c}} \tilde{u}_{0 \mathrm{c}}^{\prime} \hat{y}}\left(\hat{T}_{1 / 2}\right)_{\hat{y} \hat{y}}
\end{aligned}
$$




$$
\begin{aligned}
\left(\hat{\Omega}_{1}+\tilde{u}_{0 \mathrm{c}}^{\prime}\right. & \left.\frac{\hat{\rho}_{1}}{\tilde{\rho}_{0 \mathrm{c}}}-\frac{\hat{\psi}_{1 / 2}}{\tilde{\rho}_{0 \mathrm{c}} \tilde{u}_{0 \mathrm{c}}^{\prime} \hat{y}}\left(\hat{\Omega}_{1 / 2}-\tilde{u}_{0 \mathrm{c}}^{\prime} \frac{\hat{T}_{1 / 2}}{\tilde{T}_{0 \mathrm{c}}}\right)_{\hat{y}}+\frac{1}{\gamma M^{2}} \frac{\hat{p}_{1}}{\tilde{\rho}_{0 \mathrm{c}} \tilde{u}_{0 \mathrm{c}}^{\prime} \hat{y}} \frac{\hat{T}_{1 / 2}\left(\hat{T}_{1 / 2}\right)_{\hat{y}}}{\tilde{T}_{0 \mathrm{c}}^{2}}\right)\left._{X}\right|_{\hat{\psi}_{0}} \\
= & \frac{1}{\gamma M^{2}} \frac{\hat{p}_{1 X}}{\tilde{u}_{0 \mathrm{c}}^{\prime} \hat{y}}\left(\hat{T}_{1}-\frac{\hat{\psi}_{1 / 2}}{\tilde{\rho}_{0 \mathrm{c}} \tilde{u}_{0 \mathrm{c}}^{\prime} \hat{y}}\left(\hat{T}_{1 / 2}\right)_{\hat{y}}\right)_{\hat{y}}-\frac{c}{\tilde{u}_{0 \mathrm{c}}^{\prime} \hat{y}}\left(\hat{\Omega}_{1 / 2}-\tilde{u}_{0 \mathrm{c}}^{\prime} \frac{\hat{T}_{1 / 2}}{\tilde{T}_{0 \mathrm{c}}}\right)_{\bar{x}} \\
& +\frac{1}{\varepsilon^{2} \delta^{2} \operatorname{Re}} \frac{\tilde{\mu}_{0 \mathrm{c}}}{\tilde{\rho}_{0 \mathrm{c}} \tilde{u}_{0 \mathrm{c}}^{\prime} \hat{y}}\left(\hat{\Omega}_{1 / 2}\right)_{\hat{y} \hat{y}}
\end{aligned}
$$

where the notation on the left-hand sides indicates differentiation with $\hat{\psi}_{0}$ fixed, and so these terms represent derivatives along the approximate streamlines. Since the quantities $\hat{T}_{1}$ and $\hat{\Omega}_{1}$ appear only in the combinations $\hat{T}_{1}-\hat{\psi}_{1 / 2}\left(\hat{T}_{1 / 2}\right)_{\hat{\psi}_{0}}$ and $\hat{\Omega}_{1}-\hat{\psi}_{1 / 2}\left(\hat{\Omega}_{1 / 2}\right)_{\hat{\psi}_{0}}$, the solutions for $\hat{T}_{1}$ and $\hat{\Omega}_{1}$ contain terms $\hat{\psi}_{1 / 2}\left(\hat{T}_{1 / 2}\right)_{\hat{\psi}_{0}}$ and $\hat{\psi}_{1 / 2}\left(\hat{\Omega}_{1 / 2}\right)_{\hat{\psi}}$, respectively. Thus the quantities $\hat{\Theta}_{1 / 2 \pm}$ and $\hat{Q}_{1 / 2 \pm}$ can be thought of as functions of $\hat{\psi}=\hat{\psi}_{0}+\varepsilon^{1 / 2} \hat{\psi}_{1 / 2}+\cdots$ rather than $\hat{\psi}_{0}$, and the terms mentioned (with a factor $\varepsilon^{1 / 2}$ ) are then the linear terms in Taylor expansions of $\hat{\Theta}_{1 / 2 \pm}(\hat{\psi}, \bar{x})$ or $\hat{Q}_{1 / 2 \pm}(\hat{\psi}, \bar{x})$ about $\hat{\psi}=\hat{\psi}_{0}$. A reformulation of the entire critical-layer problem in terms of $\hat{\psi}$, however, does not seem to offer any substantial advantage, and the present formulation in terms of $X, \hat{y}$, and $\bar{x}$ is therefore retained.

The order of the Reynolds number Re $\gg 1$, still unspecified in terms of the small parameters $\varepsilon$ and $\delta$, is chosen at this point such that the diffusion terms are of the same order as the other terms appearing in (5.20) and (5.21). Since $\delta$ and $\varepsilon$ have been taken to be of the same order in (2.1), the choice for Re can be expressed by

$$
\operatorname{Re}^{-1}=O\left(\delta^{4}\right)
$$

and then also $\left(\varepsilon^{2} \delta^{2} \mathrm{Re}\right)^{-1}=O(1)$ in (5.20) and (5.21). The choices (2.1) and (5.22) specify the particular limiting case $\varepsilon \rightarrow 0, \delta \rightarrow 0, \operatorname{Re} \rightarrow \infty$ with $\varepsilon / \delta$ and $\delta^{4} \mathrm{Re}$ held fixed, for which all the physical effects represented in (5.20) and (5.21) are retained at the same level of approximation.

Bearing in mind the expansions (5.4) and (5.6) for $T$ and $\Omega$, one can see that the ratio of diffusion terms to convection terms in the critical layer is $O\left[\left(\varepsilon^{3 / 2} \delta^{2} \mathrm{Re}\right)^{-1}\right]$, which tends to zero in the present limit with $\delta^{4} \mathrm{Re}$ and $\varepsilon / \delta$ held fixed. The significance of this ratio was pointed out by Benney and Bergeron [12] and explored in further detail by Haberman [13]; the critical layer is inviscid and nonlinear, or viscous and linear, when the parameter $\lambda=\left(\varepsilon^{3 / 2} \delta^{2} \mathrm{Re}\right)^{-1}$ is small or large, respectively. (If the reference length were the shear-layer thickness instead of the spatial period, as in $[12,13]$, the factor $\delta^{2}$ would be absent in the definition of $\lambda$.) The present case, for small 
values of $\lambda$, is a different limiting case from that of $[6,7]$, in which $\lambda$ is held fixed in the limit and the amplitude is taken to be $\varepsilon=O\left(\delta^{2}\right)$. The critical layer is thinner in [6,7], with $\tilde{y}=O(\delta)$ there, and terms representing effects of diffusion and slow distortion appear in the first approximation. The result is a pair of nonlinear partial differential equations in the fast and slow longitudinal variables and the transverse critical-layer coordinate. In the present case the complete problem formulation for the largest perturbations requires consideration of the second approximation as well.

The additional conditions needed for the determination of $\hat{\Theta}_{1 / 2 \pm}$ and $\hat{Q}_{1 / 2 \pm}$ in (5.15), (5.16) take the form of statements about integrals of the right-hand sides of (5.20) and (5.21). Since the left-hand sides of these equations are derivatives along streamlines, integrals with respect to $X$ along streamline segments having the same values of $\hat{T}_{1}$ and $\hat{\Omega}_{1}$ at both end points are zero; the right-hand sides then also have zero integrals along the same paths. Outside the cat's-eye streamlines, for $\hat{\psi}_{0}>0$, the solution is required to be periodic in $X$. It follows that the integrals over one period must be zero. Since lengths have been made nondimensional with a spatial period of the disturbance, the integrations may be taken, e.g., from $X=0$ to $X=1$. For the closed streamlines inside the cat's eyes, it is required that $\hat{T}_{1}$ and $\hat{\Omega}_{1}$ be single valued, and therefore the integrals around any closed streamline must be zero. It can be seen that the right-hand sides of (5.20) and (5.21) are odd functions of $\hat{y}$. This follows because $\hat{\Theta}_{1 / 2 \pm}$ and $\hat{Q}_{1 / 2 \pm}$ are functions of $\hat{\psi}_{0}$, and $\hat{\psi}_{0}$ is an even function of $\hat{y}$; that the first term on the right side of (5.21) is odd follows from (5.20). From symmetry considerations, then, an integral over the upper half of a closed streamline is just one-half the integral around the entire curve. Thus, not only are the integrals of (5.20) and (5.21) equal to zero around a closed streamline, but also are zero separately over the upper and lower halves of the streamline.

The conditions for $\hat{\psi}_{0}>0$ and $\hat{\psi}_{0}<0$ can be written in a single form if integration limits denoted by $X_{0}$ and $X_{1}$ are functions of $\hat{\psi}_{0}$ having different definitions for $\hat{\psi}_{0}>0$ and $\hat{\psi}_{0}<0$. Outside the cat's eyes, the integrations are to be taken over one period, and so we may take

$$
X_{0}\left(\hat{\psi}_{0}\right)=0, \quad X_{1}\left(\hat{\psi}_{0}\right)=1 \quad \text { when } \hat{\psi}_{0}>0
$$

For the closed streamlines, $X_{0}$ and $X_{1}$ are such that $\hat{\psi}_{00}=\hat{\psi}_{0}$ at these values of $X$. The definitions are then expressed in the implicit form

$$
\hat{\psi}_{00}\left(X_{0}, \bar{x}\right)=\hat{\psi}_{0}, \quad \hat{\psi}_{00}\left(X_{1}, \bar{x}\right)=\hat{\psi}_{0} \quad \text { when } \hat{\psi}_{0}<0
$$

where we take $0<X_{0}<X_{1}<1$. 
Since the integrals of the left-hand sides of (5.20) and (5.21) are necessarily zero, the required conditions are expressed by setting the integrals of the right-hand sides equal to zero:

$$
\begin{aligned}
0= & -c \int_{X_{0}}^{X_{1}} \frac{\left(\hat{T}_{1 / 2}\right)_{\bar{x}}}{\tilde{u}_{0 c}^{\prime} \hat{y}} d X+\frac{1}{\varepsilon^{2} \delta^{2} \operatorname{Re} \operatorname{Pr}} \frac{\tilde{\mu}_{0 \mathrm{c}}}{\tilde{\rho}_{0 \mathrm{c}}} \int_{X_{0}}^{X_{1}} \frac{1}{\tilde{u}_{0 \mathrm{c}}^{\prime} \hat{y}}\left(\hat{T}_{1 / 2}\right)_{\hat{y} \hat{y}} d X \\
0= & \frac{1}{\gamma M^{2}} \int_{X_{0}}^{X_{1}} \frac{\hat{p}_{1 X}}{\tilde{u}_{0 \mathrm{c}}^{\prime} \hat{y}}\left(\hat{T}_{1}-\frac{\hat{\psi}_{1 / 2}}{\tilde{\rho}_{0 \mathrm{c}} \tilde{u}_{0 \mathrm{c}}^{\prime} \hat{y}}\left(\hat{T}_{1 / 2}\right)_{\hat{y}}\right)_{\hat{y}} d X \\
& -\int_{X_{0}}^{X_{1}} \frac{c}{\tilde{u}_{0 \mathrm{c}}^{\prime} \hat{y}}\left(\hat{\Omega}_{1 / 2}-\tilde{u}_{0 \mathrm{c}}^{\prime} \frac{\hat{T}_{1 / 2}}{\tilde{T}_{0 \mathrm{c}}}\right)_{\bar{x}} d X \\
& +\frac{1}{\varepsilon^{2} \delta^{2} \operatorname{Re}} \frac{\tilde{\mu}_{0 \mathrm{c}}}{\tilde{\rho}_{0 \mathrm{c}}} \int_{X_{0}}^{X_{1}} \frac{1}{\tilde{u}_{0 \mathrm{c}}^{\prime} \hat{y}}\left(\hat{\Omega}_{1 / 2}\right)_{\hat{y} \hat{y}} d X
\end{aligned}
$$

where the integrands are understood to be evaluated along streamlines. Each of the equations (5.25) and (5.26) contains terms representing effects of diffusion and of slow variation; the vorticity equation (5.26) also contains an additional integral associated with the baroclinic torque, which can be rewritten in terms of $\hat{T}_{1 / 2}$ and $\hat{p}_{1}$ with the help of (5.20).

A result of this kind was given in another context by Goldstein and Hultgren [14], for a stage in the downstream development of disturbances to an incompressible shear layer when the appropriate slow variable becomes large. The present formulation is a generalization of that given by Goldstein and Hultgren, but in terms of parameter expansions rather than coordinate expansions, and with the temperature as an additional dependent variable.

For streamlines near the centers of the cat's eyes, the diffusion terms in (5.25) and (5.26) can be approximated by substituting (5.12), (5.15), and (5.16), with $\hat{\psi}_{00}$ expanded in Taylor series about the value of $X$ at the center, where $\hat{\psi}_{00}$ has its minimum value $\hat{\psi}_{00 \mathrm{~m}}$. If just the constant and the quadratic term are retained in each case, the integrations over $X$ can be carried out, giving differential equations in the variables $\hat{\psi}_{0}$ and $\bar{x}$ that describe the local flow behavior. After this is done, (5.25) becomes

$$
\left.-\left(\hat{\Theta}_{1 / 2 \pm}\right)_{\bar{x}}+\text { (const. }\right)\left(\left(\hat{\psi}_{0}-\hat{\psi}_{00 \mathrm{~m}}\right)\left(\hat{\Theta}_{1 / 2 \pm}\right)_{\hat{\psi}_{0}}\right)_{\hat{\psi}_{0}}+\cdots=0
$$

where the derivatives of $\hat{\Theta}_{1 / 2 \pm}$ are evaluated at the center of a cat's eye; a corresponding equation is found from (5.26), with the help of (5.20). The results show that one solution for each of $\hat{\Theta}_{1 / 2 \pm}$ and $\hat{Q}_{1 / 2 \pm}$ has a logarith- 
mic form as $\hat{\psi}_{0}$ approaches its value at the center. Since derivatives with respect to $\hat{\psi}_{0}$ become larger here, it is to be expected that there will be a small region in which the diffusion terms should no longer be neglected in comparison with the convection terms and that the solution to an appropriate new set of limiting equations in this region could serve to remove the singular behavior. Goldstein and Hultgren [14] show this to be the case for incompressible flow and conclude that the logarithmic behavior is not sufficient to justify omission of the singular solution. Instead they derive an integral condition, a "generalized Prandtl-Batchelor theorem," which shows that the vorticity is not constant inside the cat's eyes and which excludes the singular solution since the integration constant multiplying this solution is found to be zero.

In the present case, supplementary integral conditions for the flow inside the cat's eyes are found by following a procedure quite similar to that of [14]. The differential equations (5.17) and (5.18) for $\hat{T}_{1}$ and $\hat{\Omega}_{1}$ can be regarded as vector equations, with convection terms in the form $\left(\tilde{u}_{0 \mathrm{c}}^{\prime} \hat{y} f\right)_{X}+\left(\hat{V}_{2} f\right)_{Y}$ each considered as the curl of a vector normal to the streamlines $d \hat{y} / d X=$ $\hat{V}_{2} /\left(\tilde{u}_{0 \mathrm{c}}^{\prime} \hat{y}\right)$; the terms involving $\hat{u}_{1}$ and $\hat{V}_{5 / 2}$ are rewritten in the desired form with the help of (5.19). The line integrals of these vectors around closed streamlines are of course zero, and by Stokes' theorem (or Green's theorem in the plane) the area integrals of the remaining terms are zero; these latter terms are the same as those on the right-hand sides of (5.20) and (5.21), except for the factor $\tilde{u}_{0 c}^{\prime} \hat{y}$. For each closed streamline, the results can be expressed in terms of line integrals around that streamline:

$$
\begin{aligned}
& 0=\oint\left\{-c \int_{0}^{\hat{y}}\left(\hat{T}_{1 / 2}\right)_{\bar{x}} d \hat{y}+\frac{1}{\varepsilon^{2} \delta^{2} \operatorname{RePr}} \frac{\tilde{\mu}_{0 \mathrm{c}}}{\tilde{\rho}_{0 \mathrm{c}}}\left(\hat{T}_{1 / 2}\right)_{\hat{y}}\right\} d X \\
& 0=\oint\left\{\frac{1}{\gamma M^{2}} \hat{p}_{1 X}\left(\hat{T}_{1}-\frac{\hat{\psi}_{1 / 2}}{\tilde{\rho}_{0 \mathrm{c}} \tilde{u}_{0 \mathrm{c}}^{\prime} \hat{y}}\left(\hat{T}_{1 / 2}\right)_{\hat{y}}\right)-c \int_{0}^{\hat{y}}\left(\hat{\Omega}_{1 / 2}-\tilde{u}_{0 \mathrm{c}}^{\prime} \frac{\hat{T}_{1 / 2}}{\tilde{T}_{0 \mathrm{c}}}\right)_{\bar{x}} d \hat{y}\right. \\
& \left.+\frac{1}{\varepsilon^{2} \delta^{2} \operatorname{Re}} \frac{\tilde{\mu}_{0 c}}{\tilde{\rho}_{0 \mathrm{c}}}\left(\hat{\Omega}_{1 / 2}\right)_{\hat{y}}\right\} d X
\end{aligned}
$$

where, of course, $\hat{y}>0$ and $\hat{y}<0$ for the upper and lower parts of a streamline, respectively, and the first integral in (5.29) can be rewritten with the help of (5.20).

The conditions (5.28) and (5.29) are analogous to the condition obtained in [14] for the vorticity in the incompressible case. With a coordinate change from $X, \hat{y}$ to $X, \hat{\psi}_{0}$ the area element is transformed from $d X d \hat{y}$ to $\left(\tilde{\rho}_{0 \mathrm{c}} \tilde{u}_{0 \mathrm{c}}^{\prime} \hat{y}\right)^{-1} d X d \hat{\psi}_{0}$, and, as for the vorticity equation in [14], the conditions (5.28) and (5.29), except for a constant factor, can be thought of as integrals 
over $\hat{\psi}_{0}$ of Eqs. (5.25) and (5.26). Again as in [14], (5.28) and (5.29) provide additional information about the behavior of the solutions near the center of a cat's eye. By expanding $\hat{\psi}_{00}$ in Taylor series about the value of $X$ at the center, we can approximate (5.28) and (5.29) for streamlines close to the center. As $\hat{\psi}_{0} \rightarrow \hat{\psi}_{00 \mathrm{~m}}$, (5.28) leads to a relation between the values of $\left(\hat{\Theta}_{1 / 2 \pm}\right)_{\bar{x}}$ and $\left(\hat{\Theta}_{1 / 2 \pm}\right)_{\hat{\psi}_{0}}$ at the center, indicating that $\left(\hat{\Theta}_{1 / 2 \pm}\right)_{\hat{\psi}_{0}}$ is bounded there, and a corresponding result for $\hat{Q}_{1 / 2 \pm}$ follows from (5.29). Thus it is found that the conditions (5.28) and (5.29) require the absence of logarithmic terms in $\hat{\Theta}_{1 / 2 \pm}$ and $\hat{Q}_{1 / 2 \pm}$ as $\hat{\psi}_{0}$ approaches its value at the center and, moreover, that the derivatives $\left(\hat{\Theta}_{1 / 2 \pm}\right)_{\hat{\psi}_{0}}$ and $\left(\hat{Q}_{1 / 2 \pm}\right)_{\hat{\psi}_{0}}$ remain finite as $\hat{\psi}_{0} \rightarrow \hat{\psi}_{00 \mathrm{~m}}$. These conclusions represent the new information that is contained in (5.28) and (5.29) but not in (5.25) and (5.26). The situation is analogous to that in [14], where, as already noted, the additional condition shows that an integration constant multiplying a logarithmic term must be zero.

Throughout the regions with closed streamlines, the temperature as well as the vorticity is not constant, since, as disturbances move downstream, diffusion effects have acted for a limited period of time that is not sufficient to allow the development of an equilibrium state for these quantities. The transverse spatial scale for spreading (in dimensional form, the square root of the product of kinematic viscosity and time) is the same as the scale for the critical-layer thickness, and so $\hat{\Theta}_{1 / 2 \pm}$ and $\hat{Q}_{1 / 2 \pm}$ are still nonuniform in the region considered, for $\bar{x}=O(1)$.

\subsection{Evolution equation}

To obtain an evolution equation for $A_{1}$, it is necessary to match the expressions obtained for the velocity $u$ in the main shear layer and in the critical layer, and thus to obtain another expression for the difference $G_{2+}-G_{2-}$, which can be combined with that given by (4.23). This in turn requires knowing the form of $\hat{\Theta}_{1 / 2 \pm}$ and $\hat{Q}_{1 / 2 \pm}$ as $\hat{\psi}_{0} \rightarrow \infty$.

As $\tilde{y} \rightarrow 0$, the behavior of the solutions for $T$ and $\Omega$ in the main part of the shear layer is found from (4.4), (4.27), and (4.30). If it is assumed that direct matching is possible, the leading terms in the expansions of the critical-layer solutions for $\hat{\Theta}_{1 / 2 \pm}$ and $\hat{Q}_{1 / 2 \pm}$ as $\hat{y} \rightarrow \pm \infty$ are found from (5.15) and (5.16) as

$$
\begin{aligned}
& \hat{\Theta}_{1 / 2 \pm}= \pm \frac{\tilde{T}_{0 \mathrm{c}}^{\prime}}{\tilde{T}_{0 \mathrm{c}}}\left(\frac{2}{\tilde{\rho}_{0 \mathrm{c}} \tilde{u}_{0 \mathrm{c}}^{\prime}}\right)^{1 / 2} \hat{\psi}_{0}^{1 / 2}\left(1+\frac{\Gamma\left(A_{1 X}\right)_{\mathrm{m}}}{2 \tilde{u}_{0 \mathrm{c}}^{\prime}} \frac{1}{\hat{\psi}_{0}}+\cdots\right) \\
& \hat{Q}_{1 / 2 \pm}=\mp \tilde{u}_{0 \mathrm{c}}^{\prime \prime}\left(\frac{2}{\tilde{\rho}_{0 \mathrm{c}} \tilde{u}_{0 \mathrm{c}}^{\prime}}\right)^{1 / 2} \hat{\psi}_{0}^{1 / 2}\left(1+\frac{\Gamma\left(A_{1 X}\right)_{\mathrm{m}}}{2 \tilde{u}_{0 \mathrm{c}}^{\prime}} \frac{1}{\hat{\psi}_{0}}+\cdots\right),
\end{aligned}
$$


where $\hat{y}$ has been related to $\hat{\psi}_{0}$ by $(5.12)$ and we have expanded $\left(\hat{\psi}_{0}-\hat{\psi}_{00}\right)^{1 / 2}$ for large $\hat{\psi}_{0}$. This proposed direct matching with solutions in the main shear layer does not contradict (5.25) and (5.26); it is assumed here that no additional terms of order $\hat{\psi}_{0}^{-1 / 2}$ or larger are needed in (5.30) and (5.31) and that the matching can be carried out as proposed. That is, in contrast to [14], there appears to be no need for an additional flow region between the main shear layer and the critical layer.

In the critical layer, integration of the terms $O\left(\varepsilon^{1 / 2} / \delta\right)$ in the definition (2.12) for the vorticity gives

$$
\hat{u}_{1}=\mp \frac{1}{\left(2 \tilde{\rho}_{0 \mathrm{c}} \tilde{u}_{0 \mathrm{c}}^{\prime}\right)^{1 / 2}} \int_{\hat{\psi}_{00}}^{\hat{\psi}_{0}}\left(\Gamma A_{1 X}\left(\hat{\Theta}_{1 / 2 \pm}\right)_{\hat{\psi}_{0}}+\hat{Q}_{1 / 2 \pm}\left(\hat{\psi}_{0}, \bar{x}\right)\right) \frac{d \hat{\psi}_{0}}{\left(\hat{\psi}_{0}-\hat{\psi}_{00}\right)^{1 / 2}}
$$

where the lower integration limit is chosen so that $\hat{u}_{1}=0$ when $\hat{y}=0$, consistent with the definition given earlier for the line $y=A$ as the line where $u-c$ is zero. With the help of (5.30) and (5.31), we can now calculate the largest terms in $\hat{u}_{1}$ as $\hat{y} \rightarrow \pm \infty$. After addition and subtraction of $\tilde{u}_{0 \mathrm{c}}^{\prime \prime} \hat{y}$ inside the brackets in the integrand of (5.32), the expansion (5.1) for $u$ gives, as $\hat{y} \rightarrow \pm \infty$,

$$
\begin{aligned}
u-c= & \varepsilon^{1 / 2} \tilde{u}_{0 c}^{\prime} \hat{y}+\varepsilon \frac{1}{2} \tilde{u}_{0 c}^{\prime \prime} \hat{y}^{2}+\cdots+\varepsilon \frac{\Gamma A_{1 X}}{2 \tilde{\rho}_{0 c} \tilde{u}_{0 c}^{\prime}}\left(\frac{\tilde{u}_{0 c}^{\prime \prime}}{\tilde{u}_{0 c}^{\prime}}-\frac{\tilde{T}_{0 c}^{\prime}}{\tilde{T}_{0 c}}\right) \ln \left(\frac{1}{2} \tilde{\rho}_{0 c} \tilde{u}_{0 c}^{\prime} \hat{y}^{2}\right) \\
& +\varepsilon \tilde{u}_{0 c}^{\prime} H_{ \pm} A_{1 X}+\varepsilon \tilde{u}_{0 c}^{\prime} I_{ \pm}+\cdots
\end{aligned}
$$

where $H_{ \pm}$and $I_{ \pm}$are defined by

$$
\begin{aligned}
& H_{ \pm}=\mp \frac{\Gamma}{\left(2 \tilde{\rho}_{0 \mathrm{c}} \tilde{u}_{0 \mathrm{c}}^{\prime}\right)^{1 / 2}} f_{\hat{\psi}_{00}}^{\infty} \frac{\left(\hat{\Theta}_{1 / 2 \pm}\right)_{\hat{\psi}_{0}}}{\tilde{u}_{0 \mathrm{c}}^{\prime}\left(\hat{\psi}_{0}-\hat{\psi}_{00}\right)^{1 / 2}} d \hat{\psi}_{0} \\
& I_{ \pm}=\mp \frac{1}{\left(2 \tilde{\rho}_{0 \mathrm{c}} \tilde{u}_{0 \mathrm{c}}^{\prime}\right)^{1 / 2}} f_{\hat{\psi}_{0 \mathrm{os}}}^{\infty}\left(\frac{\hat{Q}_{1 / 2 \pm}}{\tilde{u}_{0 \mathrm{c}}^{\prime}\left(\hat{\psi}_{0}-\hat{\psi}_{00}\right)^{1 / 2}} \pm\left(\frac{2}{\tilde{\rho}_{0 \mathrm{c}} \tilde{u}_{0 \mathrm{c}}^{\prime}}\right)^{1 / 2} \frac{\tilde{u}_{0 \mathrm{c}}^{\prime \prime}}{\tilde{u}_{0 \mathrm{c}}^{\prime}}\right) d \hat{\psi}_{0}
\end{aligned}
$$

and are functions of $X$ and $\bar{x}$. The notation is intended to indicate that the finite part should be taken; the need for this notation is seen to disappear in the evolution equation for $A_{1}$, since singular terms cancel. 
Matching with the expansion of (4.1) as $\tilde{y} \rightarrow 0$, using the expansion (4.26) for $\tilde{u}_{1}$ and setting $\hat{y}=\tilde{y} / \varepsilon^{1 / 2}$, leads to an expression for $G_{2 \pm}$ :

$$
\begin{aligned}
G_{2 \pm}= & -\frac{\Gamma A_{1 X}}{2 \tilde{\rho}_{0 \mathrm{c}} \tilde{u}_{0 \mathrm{c}}^{\prime 2}}\left(\frac{\tilde{u}_{0 \mathrm{c}}^{\prime \prime}}{\tilde{u}_{0 \mathrm{c}}^{\prime}}-\frac{\tilde{T}_{0 \mathrm{c}}^{\prime}}{\tilde{T}_{0 \mathrm{c}}}\right) \ln \left(\frac{\tilde{\rho}_{0 \mathrm{c}} \tilde{u}_{0 \mathrm{c}}^{\prime}}{2 \varepsilon}\right)-J_{ \pm} A_{1 X} \\
& +\frac{\Gamma A_{1 X}}{\tilde{\rho}_{0 \mathrm{c}} \tilde{u}_{0 \mathrm{c}}^{\prime 2}}\left(\frac{3 \tilde{u}_{0 \mathrm{c}}^{\prime \prime}}{2 \tilde{u}_{0 \mathrm{c}}^{\prime}}-\frac{\tilde{T}_{0 \mathrm{c}}^{\prime}}{\tilde{T}_{0 \mathrm{c}}}\right)-H_{ \pm} A_{1 X}-I_{ \pm}
\end{aligned}
$$

Thus, as anticipated, the functions $G_{2 \pm}$, and therefore also the solutions for $\tilde{V}_{2}, \tilde{u}_{1}$, and $\tilde{T}_{1}$ given by (4.14), (4.16), and (4.17), each contain a term with a factor $\ln \varepsilon$. From (4.20) it follows that the functions $F_{2 \pm}$ include such a term, and therefore each of the perturbations in the external flow will have a term proportional to $\ln \varepsilon$. On the other hand, (5.32) shows that there is no term of this kind in $\hat{u}_{1}$; the other solutions for the critical layer likewise have no term containing $\ln \varepsilon$.

The result (5.36) can now be combined with (4.23) and (5.9), which were obtained from the matching conditions for $v$ and $p$. The result is an evolution equation for the displacement function $A_{1}(X, \bar{x})$ :

$$
\begin{aligned}
\left(C_{+}-C_{-}\right) A_{1 \bar{x}}= & \left(\beta_{+}-\beta_{-}\right)\left(1-\frac{\gamma+1}{4} \Gamma^{2} M^{4}\right) A_{1 X}^{2} \\
& -\frac{\delta}{\varepsilon}\left\{\left(J_{+}-J_{-}\right) A_{1 X}+\left(K_{+}-K_{-}\right) A_{1 X}\right. \\
& \left.+\left(H_{+}-H_{-}\right) A_{1 X}+\left(I_{+}-I_{-}\right)\right\}_{X} .
\end{aligned}
$$

The integrals $H_{ \pm}$and $I_{ \pm}$, given by (5.34) and (5.35), are functions of $X$ and $\bar{x}$, since they depend on the disturbed temperature and velocity profiles in the critical layer, through $\hat{\Theta}_{1 / 2 \pm}$ and $\hat{Q}_{1 / 2 \pm}$, and on the disturbed pressure, through $\hat{\psi}_{00}$ and therefore $A_{1 X}$ and $p_{1}$, according to (5.8) and (5.12). The integrals $J_{ \pm}$and $K_{ \pm}$, defined in (4.28) and (4.29), depend only on the undisturbed velocity and temperature profiles $\tilde{u}_{0}$ and $\tilde{T}_{0}=1 / \tilde{\rho}_{0}$ in the main part of the shear layer, and are thus independent of both $X$ and $\bar{x}$. The terms containing these integrals also appear in the evolution equation obtained in [7], but with a somewhat different treatment of the singular integrand, as noted earlier. The term on the left-hand side of (5.37) likewise is the same as that found in [7], whereas the term proportional to $A_{1 X}^{2}$ arises from nonlinearity in the external flow and is absent in [7] because a smaller 
amplitude is considered there. Each of the integrals $H_{ \pm}, I_{ \pm}, J_{ \pm}$, and $K_{ \pm}$ appears in a linear way in the velocity $u$, and (5.37) is obtained by matching solutions for $u$; thus no products of integrals appear in (5.37). The integrands of $\left(H_{+}-H_{-}\right)$and $\left(I_{+}-I_{-}\right)$involve the combinations $\left(\hat{\Theta}_{1 / 2+}\right)_{\hat{\psi}_{0}}+$ $\left(\hat{\Theta}_{1 / 2-}\right)_{\psi_{0}}$ and $\hat{Q}_{1 / 2+}+\hat{Q}_{1 / 2-}$. According to (5.30) and (5.31), each of these sums is smaller than $O\left(\hat{\psi}_{0}^{-1 / 2}\right)$ as $\hat{\psi}_{0} \rightarrow \infty$; thus the integrals $\left(H_{+}-H_{-}\right)$and $\left(I_{+}-I_{-}\right)$exist. These terms in (5.37) arise from integration of the vorticity across the critical layer and differ from the corresponding terms in [7] because the critical-layer solutions are different.

The evolution equation (5.37) for the displacement function $A_{1}$ is the main result of the derivation. Its solution requires an initial condition that specifies $A_{1}$ for one period in $X$ at a large negative value of $\bar{x}$. The integrals in (5.37) involve the functions $\hat{\Theta}_{1 / 2 \pm}$ and $\hat{Q}_{1 / 2 \pm}$, and so Eqs. (5.25) and (5.26), which contain $A_{1}$ as well as $\hat{\Theta}_{1 / 2 \pm}$ and $\hat{Q}_{1 / 2 \pm}$, must be solved simultaneously with (5.37). Initial and boundary conditions for $\hat{\Theta}_{1 / 2 \pm}$ and $\hat{Q}_{1 / 2 \pm}$ are also needed. It is expected that these functions will approach zero as $\bar{x} \rightarrow-\infty$; the manner in which they might approach zero is considered briefly below. The forms of $\hat{\Theta}_{1 / 2 \pm}$ and $\hat{Q}_{1 / 2 \pm}$ as $\hat{\psi}_{0} \rightarrow \pm \infty$ are given by (5.30) and (5.31). For the closed streamlines inside the cat's eyes, the integral conditions (5.28) and (5.29) must be satisfied; i.e., $\left(\hat{\Theta}_{1 / 2+}\right)_{\hat{\psi}_{0}}$ and $\left(\hat{\Theta}_{1 / 2-}\right)_{\hat{\psi}_{0}}$ remain bounded as the cat's-eye centers are approached. As in the analogous incompressible problem, the vorticity, and now also the temperature, is presumed to be continuous at the boundaries $\hat{\psi}_{0}=0$ of the cat's eyes, but the derivatives $\left(\hat{\Theta}_{1 / 2+}\right)_{\hat{\psi}_{0}}$ and $\left(\hat{\Theta}_{1 / 2-}\right)_{\hat{\psi}_{0}}$ might be expected to have jump discontinuities there. For a numerical solution, it may be necessary, as in [14], to study the thin viscous layers about $\hat{\psi}_{0}=0$, following Brown and Stewartson [15]; this is not done here.

Further upstream, in terms of the slow variable $\bar{x}$, it is expected that the amplitude is smaller, and so $A_{1}$ is smaller. From the expression (5.12) for $\hat{\psi}_{0}$, the critical layer should be thinner, with values of $\hat{y}^{2}$ of the same order as a typical value of $A_{1}$. From matching with the temperature and vorticity in the main part of the boundary layer, the values of $\hat{\Theta}_{1 / 2 \pm}$ and $\hat{Q}_{1 / 2 \pm}$ will presumably decrease as $A_{1}^{1 / 2}$. If these suppositions are all correct, then the integrals $J_{+}-J_{-}$and $K_{+}-K_{-}$across the main part of the boundary layer and $H_{+}-H_{-}$and $I_{+}-I_{-}$across the critical layer will all remain in the evolution equation (5.37), but the nonlinear term proportional to $A_{1 X}^{2}$ will be absent in the leading approximation. Since the integrals that remain will again lead to slow changes on the scale of the $\bar{x}$ coordinate, it is necessary that the derivative $A_{1 \bar{x}}$ remain in the equation while $A_{1}$ decreases. A power-law decay of $A_{1}$ would not permit the needed balance of terms, whereas for an exponential decrease $A_{1}$ and $A_{1 \bar{x}}$ would change at the same rate as $\bar{x} \rightarrow-\infty$. Thus it appears that the upstream decay of $A_{1}$ will be exponential in $\bar{x}$; this is noted again in Section 6 . 
The small parameters $\varepsilon, \delta$, and $\mathrm{Re}^{-1}$ enter the problem formulation in the combinations $\varepsilon / \delta$ and $\delta^{4} \mathrm{Re}$, which may be thought of as similarity parameters for this particular limiting case. The result (5.37) for the evolution equation, as well as the solutions for the vorticity and temperature, may, however, not be uniformly valid for large or small values of these similarity parameters. Any such nonuniformity implies the need for a different problem formulation in another special limiting case corresponding to a different pair of order relations among $\varepsilon, \delta$, and $\mathrm{Re}^{-1}$. To identify other special limiting cases, it may be convenient to replace the shear-layer thickness $\delta$ (nondimensional with the spatial period) in terms of the Reynolds number Re and the nondimensional distance $L$ from the origin of the shear layer. If the shear layer is regarded as having zero initial thickness at some upstream location, a self-similar solution is available, and the thickness is known to grow as the square root of the distance. Thus we may set

$$
\delta=L^{1 / 2} \operatorname{Re}^{-1 / 2}
$$

We might then think of taking $1 / L$ instead of $\delta$ as one of the basic small parameters. If, for example, the viscous length and the wavelength are regarded as specified, the choice of a relation between $\delta$ and $1 / \operatorname{Re}$ is equivalent to a statement about the distance $L$. That is, if $\operatorname{Re}$ is specified, we can think of various possible choices for the position $L$ and the nondimensional amplitude $\varepsilon$. The results obtained thus far are based on the assumption that $L=O\left(\mathrm{Re}^{1 / 2}\right)$, with $\varepsilon$ and $\delta$ of order $\mathrm{Re}^{-1 / 4}$. Some suggestions are made in the next section concerning the nature of the local solutions when different choices are made for the orders of $\varepsilon$ and $\delta$ (or $1 / L$ ).

\section{Other limiting cases}

\subsection{Vortex-sheet limit}

If a disturbance at a location where $\delta=O\left(\operatorname{Re}^{-1 / 4}\right)$ has amplitude $\varepsilon \gg \delta$, it follows from setting $\delta / \varepsilon=0$ in (5.37) that the terms depending on the velocity and temperature distributions within the shear layer disappear in the limit. Thus the shear layer appears as a vortex sheet in the first approximation, with the evolution equation given by

$$
A_{1 \bar{x}}-\frac{1}{2} \alpha A_{1 X}^{2}=0, \quad \frac{1}{2} \alpha=\frac{\beta_{+}-\beta_{-}}{C_{+}-C_{-}}\left(1-\frac{\gamma+1}{4} \Gamma^{2} M^{4}\right) .
$$

(This result can, of course, be found more directly by combining the conditions that the pressure be continuous across the vortex sheet and that 
there be no flow through the sheet.) The sign of $\alpha$ in (6.1) depends on the velocity and Mach number in the flow above and below the sheet. To illustrate the behavior of the solution we consider $\alpha>0$. One special case for which $\alpha$ is in fact positive is that in which the temperature is the same above and below, so that also $\rho_{+}=\rho_{-}=1$. For a verification that $\alpha>0$ in this case, the three factors appearing in the definition of $\alpha$ can be considered separately. First, (4.8) can be rewritten [1] as a quadratic equation for $\left\{c-\left(u_{+}+u_{-}\right) / 2\right\}^{2}$. The solution for $c$ shows that $0<u_{+}-c<c-u_{-}$and therefore (since $\beta_{+} \beta_{-}=1$ ) that $\beta_{+}<1<\beta_{-}$. Thus the relative velocity and Mach number are lower on the upper side of the sheet and higher on the lower side. Next, from the second form of the definition (4.22) for $C_{ \pm}$, with the help of the result $\beta_{+} \beta_{-}=1$ and the inequalities just noted, it can be seen that $C_{+}-C_{-}>0$ for the present case. Finally, the definition (4.7) for $\Gamma$ can be rewritten to show that $\Gamma M^{2}=\left(1+\beta_{+}^{2}\right) / \beta_{+}=\left(1+\beta_{-}^{2}\right) / \beta_{-}$. The minimum of $\left(1+\beta^{2}\right) / \beta$ occurs at $\beta=1$ and is equal to 2 , so that $\Gamma^{2} M^{4}$ is no smaller than 4 ; since $\gamma>1,1-(\gamma+1) \Gamma^{2} M^{4} / 4$ is necessarily negative. Thus $\alpha>0$ if $\rho_{+}=\rho_{-}=1$; we take $\alpha>0$ in the following without, of course, restriction to only this case.

If now (6.1) is differentiated with respect to $X$, it is seen that $A_{1 X}$ remains constant along characteristics $d X / d \bar{x}=-\alpha A_{1 X}$. The solution is given implicitly by

$$
A_{1 X}(X, \bar{x})=\vartheta\left(X+\alpha \bar{x} A_{1 X}(X, \bar{x})\right), \quad \vartheta(X)=A_{1 X}(X, 0),
$$

where $\vartheta$ is a function of a single argument and is defined in terms of its form at $\bar{x}=0$, which is considered to be known. Thus (6.2) describes the evolution of the shape of the vortex sheet in terms of an initial disturbance represented by $A_{1 X}(X, 0)$. The solution of (3.19) with the proper values at $\bar{y}=0$, as well as at $\bar{x}=0$, can then be expressed in implicit form by evaluating (6.2) at $X_{0}, \bar{x}_{0}$ and combining with (3.21). Substitution for $X_{0}$ and $\bar{x}_{0}$ from (3.20) allows representation of the argument $X_{0}+\alpha \theta \bar{x}_{0}$ in terms of $\theta$ and the coordinates $\xi, \bar{x}, \bar{y}$. Thus

$$
\theta=\vartheta(\Xi)
$$

where

$$
\Xi=\xi+\frac{(\gamma+1) \rho_{ \pm}^{2}\left(u_{ \pm}-c\right)^{4} M^{4}}{2 \beta_{ \pm}^{2}} \theta \bar{y}+\alpha\left(\bar{x}-\frac{M^{2} \rho_{ \pm} u_{ \pm}\left(u_{ \pm}-c\right)-1}{\beta_{ \pm}} \bar{y}\right) \theta
$$


Equations (6.3) and (6.4), in combination with (3.11) and (3.14), can be used to provide a representation of the complete flow field in terms of the initial surface deformation.

A simple example of a periodic shape $A_{1}(X, \bar{x})$ is provided by the initial values

$$
A_{1}(X, 0)=\cos X, \quad A_{1 X}(X, 0)=\vartheta(X)=-\sin X,
$$

where the spatial period here is taken to be $2 \pi$ rather than one. For $\bar{x}>0$, (6.2) gives the surface slope, and the curvature $A_{1 X X}(X, \bar{x})$ is found by differentiation as

$$
A_{1 X X}(X, \bar{x})=\frac{-\cos \left(X+\alpha \bar{x} A_{1 X}(X, \bar{x})\right)}{1+\alpha \bar{x} \cos \left(X+\alpha \bar{x} A_{1 X}(X, \bar{x})\right)}
$$

This expression becomes infinite when $\alpha \bar{x}=1$ and the cosine is equal to -1 , i.e., when $A_{1 X}=0$ and $\bar{x}=\alpha^{-1}$, at $X=(2 n+1) \pi$, for $n=0, \pm 1, \pm 2, \cdots$. The sheet thus undergoes a distortion, with the minima of $A_{1}$ developing corners such that weak shock waves appear for $Y>0$ and weak centered expansions for $Y<0$. The result is consistent with that of [2] for $\rho_{ \pm}=1$, with the shocks appearing on the side of the sheet where the relative flow speed $u-c$ is smaller, and the expansions appearing on the side where the speed $|u-c|$ is larger.

The flow field is found from the solution (6.3) for $\theta$, which is proportional to the local streamline slope and which for this case becomes $\theta=F_{1 \xi}=$ $-\sin \Xi$, where $\Xi$ is again defined by (6.4). Above the sheet the flow undergoes compression when the curvature is positive, i.e., in the range where $\cos \Xi<0$, while below the sheet compression occurs where $\cos \Xi>0$ and the curvature is negative. The derivative $F_{1 \xi \xi}$, proportional to the local streamline curvature, becomes infinite at the values of $\bar{y}$ where its denominator first becomes zero, for $|\cos \Xi|=1$. The singular behavior arises because the characteristics in the $X, Y$ plane are not exactly parallel, an intersection of characteristics occurring at each of the points where $F_{1 \xi \xi}$ first becomes infinite; a sketch of the wave pattern near the sheet is shown in Figure 2. Weak shock waves form at large distances above and below the sheet as a consequence of the coalescing of isentropic compression waves, at the points where characteristics first intersect. This is the far-field result for steady flow past a wavy wall, but now the "wavy wall" deforms, with increasing or decreasing curvature in portions of each spatial period that contain the minimum or maximum values of $A_{1}$, respectively. The shocks 

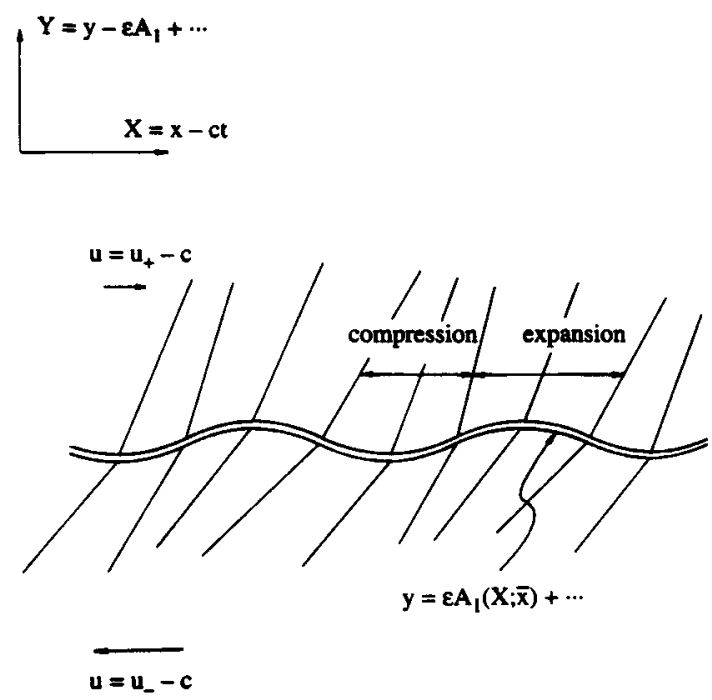

Figure 2. Disturbed shear layer for $\varepsilon \gg \delta$, with associated compression and expansion waves.

appear at $\Xi=(2 n+1) \pi$ for $\bar{y}>0$ and at $\Xi=2 n \pi$ for $\bar{y}<0$, at values of $\bar{y}$ given by

$$
\frac{(\gamma+1) \rho_{ \pm}^{2}\left(u_{ \pm}-c\right)^{4} M^{4}}{2 \beta_{ \pm}^{2}} \bar{y}+\alpha\left(\bar{x}-\frac{M^{2} \rho_{ \pm} u_{ \pm}\left(u_{ \pm}-c\right)-1}{\beta_{ \pm}} \bar{y}\right)=\frac{-1}{\cos \Xi},
$$

where the values $\cos \Xi=\mp 1$ are to be taken for $\bar{y} \gtrless 0$.

As the downstream distance $\bar{x}$ increases, the point of formation for each shock wave gradually moves downward, toward the sheet for $\bar{y}>0$ and away from the sheet for $\bar{y}<0$. When $\alpha \bar{x}=1$, shock waves reach the upper surface at the locations $X=(2 n+1) \pi$, and centered expansion waves appear at the same points on the lower surface. With further increase in downstream distance, a discontinuity in the slope of the sheet is present at $X=(2 n+1) \pi$, and the solution for $A_{1 X}$ shows that the magnitudes of these jumps continue to increase until $\alpha \bar{x}=\pi / 2$, when $\left|A_{1 X}\right|=1$ immediately ahead of and behind each jump. Thus for $1<\alpha \bar{x}<\pi / 2$ and $\bar{y}>0$, the shock waves at the surface continue to overtake, or to be overtaken by, compression waves, so that the strengths of the shocks and expansions increase at the sheet: the "kinks" at the sheet become stronger. For still larger distances, when $\alpha \bar{x}>\pi / 2$, the shocks at the surface are weakened by interaction with expansion waves, the flow turning angles at the sheet decrease, and the "kinks" become weaker. All disturbances then decay, according to this 
system of equations, as $\alpha \bar{x} \rightarrow \infty$. Although the effects of changes inside the very thin shear layer are ignored in the vortex-sheet limit, it would nonetheless be of interest to consider these higher-order effects, taking the function $A_{1 X}$ to be prescribed by the external-flow solution for a vortex sheet, modified in some way to allow for shock discontinuities; with increasing downstream distance, changes within the shear layer may possibly grow large enough that their effect on the outer flow is no longer of higher order. When the value of $\varepsilon / \delta$ is not large, the effects of nonzero thickness shown in (5.37) do of course influence the outer flow for all $\vec{x}$, but the shock-wave behavior is expected to be qualitatively similar to that in the case of a vortex sheet.

\subsection{Disturbances at other locations}

The formulation of Sections 3 through 5 is characterized by a relatively thick critical layer, with diffusion effects appearing first in a periodicity or singlevaluedness condition. It might be anticipated that some other limiting case would correspond to a different balance at a location further upstream, where the disturbance amplitude would be somewhat smaller and the critical layer somewhat thinner, so that the diffusion terms would appear earlier. In particular, a first approximation showing a balance of convection and diffusion terms in nonlinear temperature and vorticity equations might be expected.

One way to start is by considering the behavior of the evolution equation (5.37) as the slow variable $\bar{x} \rightarrow-\infty$, i.e., by moving upstream from the region discussed in previous sections. The amplitude function $A_{\mathrm{i}}$ then decreases, and the quadratic term proportional to $A_{1 X}^{2}$ is expected to become smaller in comparison with the other terms. The slow changes in amplitude now are the result of the changes in pressure and streamline slope across the main part of the shear layer and the jump in velocity across the critical layer, corresponding to the terms $O(\delta / \varepsilon)$ in (5.37). Since these terms do imply a slow growth, the slow derivative should remain in the equation, and so the slow variable originally defined in (2.2) should be redefined with scale $\delta$ rather than $\varepsilon$. Retaining the slow derivative in (5.37) as $\bar{x} \rightarrow-\infty$ also implies the functional form of $A_{1}$ as $\bar{x} \rightarrow-\infty$ and requires that $A_{1}$ decrease exponentially. However, the anticipated balance of convection and diffusion terms will occur when the amplitude is only algebraically small in terms of the parameters, and so the magnitude of $\bar{x}$ need only be logarithmically large; i.e., it is only necessary that $-\bar{x}=O(\ln \mathrm{Re})$. The slow variable should now be measured from a different reference location, say $x=x_{0}$, and redefined as $\bar{x}=\delta\left(x-x_{0}\right)$. Taking $\delta\left(x-x_{0}\right)=O(1)$ and $\delta(x-L)=$ $O(\ln \mathrm{Re})$ and recalling that $L=O\left(\mathrm{Re}^{1 / 2}\right)$ for the derivation in Section 5 , it follows that the relative change in the reference locations is given by 
$\left(L-x_{0}\right) / L=O\left(\mathrm{Re}^{-1 / 4} \ln \mathrm{Re}\right)$ and is therefore small, so that the relative change in shear-layer thickness is also small, and we again have $\delta^{4}=$ $O\left(\mathrm{Re}^{-1}\right)$ as in (5.22).

Next we can look for a possible asymptotic flow description having downstream behavior that matches with the upstream behavior found from the previous formulation. A balance between convection and diffusion shows that the critical layer appears when $\tilde{y}=O\left(\delta^{2 / 3}\right)$ rather than $O\left(\delta^{1 / 2}\right)$ as before. This is the familiar result for a viscous critical layer, as given by Lin [16]. Nonlinearity is again indicated through the need for a correction to the undisturbed stream function, as suggested by (4.34) evaluated for small $\tilde{y}$, implying a smaller order for the amplitude, namely $\varepsilon=O\left(\delta^{4 / 3}\right)$ rather than $O(\delta)$. This is then an example of a limit in which the parameter $\left(\varepsilon^{3 / 2} \delta^{2} \mathrm{Re}\right)^{-1}$ of $[12,13]$ is held fixed. Asymptotic expansions are introduced, as before, for the external flow, the main part of the shear layer, and the critical layer, with various differences appearing because of the smaller amplitude and thinner critical layer. The derivations resemble those of Sections 3 through 5 in many respects, the greatest difference arising in the now simpler formulation for the critical layer. The new representations are presumed to describe a stage in the evolution of a small disturbance that precedes the stage considered in Sections 3 through 5. It is suggested that this earlier stage exhibits nonlinearity in the critical layer but not in the external flow and that in the later stage the amplitude has grown large enough that nonlinearity enters the description of the external flow as well, giving the term proportional to $A_{1 X}^{2}$ in (5.37). Since the disturbance growth is expected to be exponential, the changes occur within the relatively small distance indicated above.

It seems convenient to express this order-of-magnitude information about the position and amplitude in terms of logarithms, defining

$$
L^{\dagger}=(\ln L) /(\ln \mathrm{Re}), \quad \varepsilon^{\dagger}=\{\ln (1 / \varepsilon)\} /(\ln \mathrm{Re})
$$

With these definitions, $L^{\dagger}$ increases as the downstream distance $L$ increases, but $\varepsilon^{\dagger}$ decreases as the amplitude $\varepsilon$ increases. Since $L=O\left(\mathrm{Re}^{1 / 2}\right)$ and, as noted, $x_{0}$ is close to $L$, the quantity $L^{\dagger}$ remains nearly constant and equal to $\frac{1}{2}$, even if $L$ is replaced by $x_{0}$, while in passing from the earlier stage to the later stage $\varepsilon^{\dagger}$ decreases from $\frac{1}{3}$ to $\frac{1}{4}$, as can be seen by substituting $\varepsilon=\delta^{4 / 3}$ in (2.1) and (5.38). Each of the two stages can then be regarded as represented by a point with coordinates $L^{\dagger}$ and $\varepsilon^{\dagger}$, namely by the points $\left(\frac{1}{2}, \frac{1}{3}\right)$ and $\left(\frac{1}{2}, \frac{1}{4}\right)$.

In the formulation given by Balsa [6, 7], different order relations were chosen among the small parameters. The choice that allowed a balance of convection and diffusion terms in the first approximation for the critical 
layer was, in the present notation, $\delta^{5} \mathrm{Re}=O(1)$ rather than $\delta^{4} \mathrm{Re}=O(1)$. From the interpretation (5.38) of $\delta$ as a measure of downstream location $L$, it follows that the present formulation describes flow disturbances at a location further upstream and with larger amplitude. In the present case, $L=O\left(\mathrm{Re}^{1 / 2}\right)$ and the amplitude is $O(\delta)$, or $O\left(\mathrm{Re}^{-1 / 4}\right)$; in [6, 7], $L=$ $O\left(\mathrm{Re}^{3 / 5}\right)$ and the amplitude is $O\left(\delta^{2}\right)$, or $O\left(\mathrm{Re}^{-2 / 5}\right)$. Thus the two representations correspond to different paths of approach to the origin in the $\varepsilon, \delta$, $\mathrm{Re}^{-1}$ parameter space. Or, in the coordinates $L^{\dagger}$ and $\varepsilon^{\dagger}$, Balsa's solution $[6,7]$ corresponds to the point $\left(\frac{3}{5}, \frac{2}{5}\right)$ rather than the present $\left(\frac{1}{2}, \frac{1}{4}\right)$ for the later stage.

We can also consider various other limits, i.e., other choices for the orders of magnitude of $\delta$ and $\varepsilon$ in terms of Re, corresponding to different points in the $L^{\dagger}, \varepsilon^{\dagger}$ plane. Each of the special points $\left(\frac{3}{5}, \frac{2}{5}\right)$ and $\left(\frac{1}{2}, \frac{1}{4}\right)$ corresponds to a problem formulation that is distinguished in the sense that the proper approximate equations at each of these points is "richer" (e.g., [17]) than the equations at any location in a small neighborhood of the point; the associated limits might be called "distinguished," in the sense defined, e.g., in [9]. It appears that only three points in the plane (for $L^{\dagger}>0$ and $\varepsilon^{\dagger}>0$ ) have this property; the third such point is identified below. There are also lines in the plane with the analogous property that along each such line the proper approximate equations are "richer" than the equations at neighboring points on either side of the line. Each of these lines represents a boundary, such that some particular effect or effects are important on one side of the line but not on the other. The special points and lines identified are shown in Figure 3.

If the amplitude is of the same order as the thickness, then $\varepsilon=O(\delta)$ and it follows, with the help of (5.38), that

$$
\varepsilon^{\dagger}=\left(1-L^{\dagger}\right) / 2
$$

Below this line $(\varepsilon \gg \delta)$ the motion is dominated by nonlinearity in the external flow, and the motion is approximated in terms of a vortex sheet, as described in Section 6.1. Above this line $(\varepsilon \ll \delta)$ nonlinearity in the external flow does not enter into the leading approximation. Clearly the point $\left(\frac{1}{2}, \frac{1}{4}\right)$ of the formulation in Section 5 lies on the line. One end point of the line is at $\left(0, \frac{1}{2}\right)$, where $L=O(1)$ and $\varepsilon=O\left(\mathrm{Re}^{-1 / 2}\right)$; here the distance $L$ has decreased to the same order as the spatial period, whereas all of the present discussion has assumed that $L$ is larger. The other end point is at $(1,0)$, where $L=O(\mathrm{Re})$ and $\varepsilon=O(1)$; here the shear-layer thickness $\delta$ has increased to the same order as the period, whereas it has been assumed throughout that $\delta$ is smaller. 


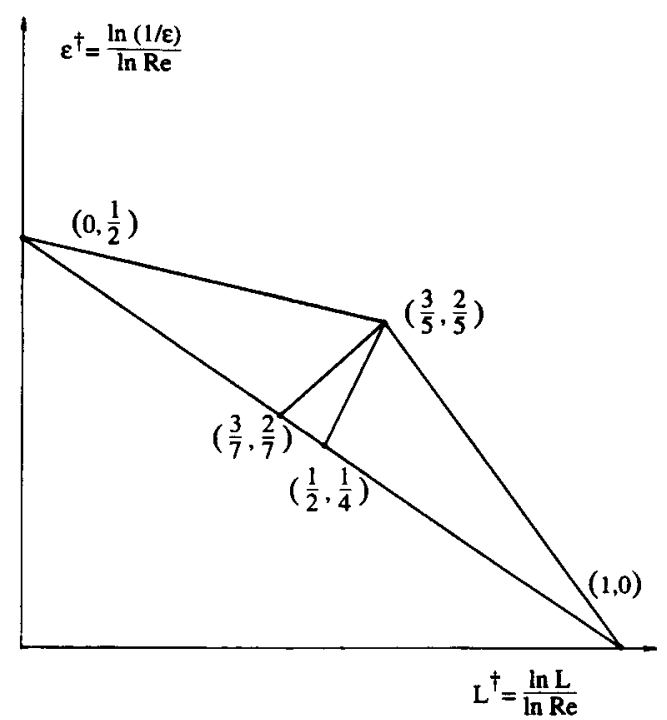

Figure 3. Identification of special limiting cases for disturbances at different locations and having different amplitudes.

The limiting forms of the equations are different on different portions of the line (6.9). For $L^{\dagger}>\frac{1}{2}$, and therefore $\delta^{4} \mathrm{Re} \rightarrow \infty$, diffusion effects are absent in (5.25) and (5.26), so that $\hat{T}_{1 / 2}$ is independent of $\bar{x}$ and $\hat{\Omega}_{1 / 2}$ varies with $\bar{x}$ only because of the term in the baroclinic torque; however, a diffusion effect is still present in thin layers bounding the cat's eyes. For $L^{\dagger}<1 / 2$, i.e., for $\delta^{4} \mathrm{Re} \rightarrow 0$, the critical-layer thickness is $O\left(\varepsilon^{1 / 2} \delta\right)$ and diffusion terms become as large as the convection terms at the point $\left(\frac{3}{7}, \frac{2}{7}\right)$, where $L=O\left(\mathrm{Re}^{3 / 7}\right)$ and $\varepsilon=O\left(\mathrm{Re}^{-2 / 7}\right)$. Since $\varepsilon=O(\delta)$, this point corresponds to a limit with $\delta^{7 / 2} \operatorname{Re}$ held fixed. For still smaller $L$, i.e., for $L^{\dagger}<\frac{3}{7}$, the energy and vorticity equations continue to show a balance of convection and diffusion terms, and the thickness of the viscous critical layer remains $O\left(\delta^{1 / 3} \mathrm{Re}^{-1 / 3}\right)$, i.e., $O\left(\delta L^{-1 / 3}\right)$. Thus, as $L$ continues to decrease, the critical-layer thickness now increases relative to the overall shear-layer thickness, and the velocity jump across the critical layer is associated with a smaller term in the critical-layer vorticity. It appears that the second terms in both temperature and vorticity are now linear in $\tilde{y}$, as in the undisturbed motion, and that the third terms are the unknown functions, matching as $|\hat{y}| \rightarrow \infty$ with terms $O(\varepsilon / \tilde{y})$ as $\tilde{y} \rightarrow 0$ in the main part of the shear layer. The evolution equation remains nonlinear because of the quadratic term and also because the integral across the critical layer will involve the displacement through the solutions for temperature and vorticity.

The point $\left(\frac{3}{7}, \frac{2}{7}\right)$ is then another special point, with nonlinearity in the external flow as well as within a quasi-equilibrium critical layer. The external 
flow and the main part of the shear layer would be described here in essentially the same way as in Sections 3 and 4 above, whereas the criticallayer equations would be similar to those of $[6,7]$, but without the slow derivative, and written in terms of a displaced coordinate $Y$ defined by (2.3). A detailed study of the equations obtained in this limit, for $\varepsilon=O(\delta)$ and $\delta=O\left(\operatorname{Re}^{-2 / 7}\right)$, might be of interest; the associated numerical problem should be considerably easier than that of solving the equations in Section 5 .

If instead the disturbance amplitude $\varepsilon$ is smaller than the overall shearlayer thickness but of the same order as the thickness of the critical layer, then two possibilities arise. A nonlinear critical layer, with relative changes in the stream function $\tilde{\psi}$ near $\tilde{y}=0$ that are not small, has thickness $O\left(\varepsilon^{1 / 2} \delta\right)$, or $O\left[(\varepsilon L / \mathrm{Re})^{1 / 2}\right]$, whereas a balance of inertia and diffusion terms for a viscous critical layer gives a thickness $O\left[\left(\delta^{2} \mathrm{Re}\right)^{-1 / 3} \delta\right]$, or $O\left(L^{1 / 6} \mathrm{Re}^{-1 / 2}\right)$. Equating the orders of the amplitude and the criticallayer thickness then gives, respectively, $\varepsilon=O\left(\delta^{2}\right)=O(L / \mathrm{Re})$ and $\varepsilon=$ $O\left(\delta^{1 / 3} \mathrm{Re}^{-1 / 3}\right)=O\left(L^{1 / 6} \mathrm{Re}^{-1 / 2}\right)$; the two estimates are the same when $\delta=O\left(\mathrm{Re}^{-1 / 5}\right)$, or $L=O\left(\mathrm{Re}^{3 / 5}\right)$. An inviscid nonlinear critical layer is found if $\delta^{5} \mathrm{Re} \rightarrow \infty$, i.e., for $L \gg \mathrm{Re}^{3 / 5}$, and a viscous critical layer if $\delta^{5} \operatorname{Re} \rightarrow 0$, i.e., for $L \ll \operatorname{Re}^{3 / 5}$. In the former case, then,

$$
\varepsilon^{\dagger}=1-L^{\dagger}
$$

and diffusion effects are expected to be absent from the differential equations describing the critical layer, but still to be present in thin layers bounding the cat's eyes. The line $(6.10)$ extends from $\left(\frac{3}{5}, \frac{2}{5}\right)$ to $(1,0)$. On the other hand, the latter case gives

$$
\varepsilon^{\dagger}=\frac{1}{2}\left(1-\frac{1}{3} L^{\dagger}\right)
$$

and the differential equations for temperature and vorticity contain a balance of convection and diffusion terms; as on the line $\varepsilon^{\dagger}=\left(1-L^{\dagger}\right) / 2$ for $0<L^{\dagger}<\frac{3}{7}$, the unknown functions appear in the third terms in the expansions. The line (6.11) extends between $\left(0, \frac{1}{2}\right)$ and $\left(\frac{3}{5}, \frac{2}{5}\right)$. It can be seen that expressions (6.10) and (6.11) coincide when $L=O\left(\mathrm{Re}^{3 / 5}\right)$ and $\varepsilon=$ $O\left(\operatorname{Re}^{-2 / 5}\right)$, which is the case in [6, 7]. The two lines (6.10) and (6.11) represent the first appearance of nonlinearity, when $\operatorname{Re}^{3 / 5} \ll L \ll \operatorname{Re}$ and $1 \ll L \ll \mathrm{Re}^{3 / 5}$ respectively: above these lines the amplitude is small enough that the disturbances are described by linear theory, but on or below these lines the critical layer is nonlinear.

At least two other line segments also correspond to systems of equations that are special in some way. The line $\varepsilon^{\dagger}=\left(3 L^{\dagger}-1\right) / 2$, where $\varepsilon / \delta \rightarrow 0$ such that $\varepsilon=O\left[\left(\delta^{3} \mathrm{Re}\right)^{-1}\right]$, connects the points $\left(\frac{1}{2}, \frac{1}{4}\right)$ and $\left(\frac{3}{5}, \frac{2}{5}\right)$. Along this line 
the formulation is similar to that of Section 5 , but the scale for $\bar{x}$ is $1 / \delta$ rather than $1 / \varepsilon$. The integrals in (5.25) and (5.26) again contain terms representing diffusion and slow variation, but $\varepsilon \ll \delta$, so that the external flow is described by linear equations and the evolution equation (5.37) for $A_{1}$ therefore no longer contains the term proportional to $A_{1 X}^{2}$. The line $\varepsilon^{\dagger}=2 L^{\dagger} / 3$ passes through $\left(\frac{3}{5}, \frac{2}{5}\right)$ and $\left(\frac{1}{2}, \frac{1}{3}\right)$ and intersects $\varepsilon^{\dagger}=\left(1-L^{\dagger}\right) / 2$ at $\left(\frac{3}{7}, \frac{2}{7}\right)$. Along the segment between $\left(\frac{3}{5}, \frac{2}{5}\right)$ and $\left(\frac{3}{7}, \frac{2}{7}\right)$ the formulation differs from that of [6,7], since $\delta^{2} \ll \varepsilon \ll \delta$ and the critical layer is a quasi-equilibrium critical layer: the critical-layer equations show a balance of convection and diffusion, but slow derivatives are absent.

The special line segments divide the plane into a number of regions within which the flow development is described by simplified equations obtained by taking suitable intermediate limits. (In the case of an incompressible shear layer, Churilov and Shukman [18] have identified ranges of parameters for which nonlinearity or viscosity or slow growth may be neglected.) In each of the examples considered above, the growth of small disturbances with increasing downstream distance is exponential and therefore occurs at a nearly constant value of $L$, i.e., along a vertical line in the $\varepsilon^{\dagger}, L^{\dagger}$ plane. In the upper part of the plane, as already noted, the disturbances are small enough to be represented in terms of the linear theory. As $\varepsilon$ increases (and $\varepsilon^{\dagger}$ decreases), one or more of the special lines is crossed until the line $\varepsilon=\delta$ is reached.

\section{Concluding remarks}

The main purpose here has been to suggest a limiting case that incorporates effects of nonzero shear-layer thickness into the description of the weakly nonlinear instability of a supersonic vortex sheet. This limit is such that nonlinearity appears in a critical layer as well as in the external flow. Thus the slow changes in amplitude are influenced not only by the wave steepening in the external flow, as in [2], but also by the changes in pressure and transverse velocity across the main part of the shear layer and the velocity jump across the critical layer, as in $[6,7]$. A set of approximate equations is derived for each of the three regions, such that asymptotic matching of the solutions should be possible. The description of the external flow reduces to that of [2] for the case considered there; effects of shear-layer structure are added in the present derivation. The perturbations in the main boundary layer are described in essentially the same way as in [7], but nonlinearity in the external flow is included here, and the formulation for the critical layer is different from that of [7], since terms representing diffusion and slow distortion now appear in the second approximation, implying a pair of integral conditions, which are coupled with an evolution equation that also differs from that of [7]. 
The equations obtained correspond to specific orders, expressed in terms of the Reynolds number based on the spatial period, for the distance from the origin of the shear layer and for the amplitude of the small disturbances, and would differ if other choices were made. The particular limiting case considered here appears to be just one of three special limiting cases. The other two correspond to a nonequilibrium viscous nonlinear critical layer, but with linear external flow, investigated in [7], or a quasi-equilibrium viscous nonlinear critical layer, with nonlinear external flow, that is mentioned briefly here. Asymptotic descriptions of the growth of small disturbances thus are characterized by different sets of approximate equations, depending on the location along the shear layer and on the disturbance amplitude.

\section{Acknowledgments}

Much of this work was carried out during visits at the Institute for Computational Mechanics in Propulsion (ICOMP), NASA Lewis Research Center. The author thanks T. F. Balsa, M. E. Goldstein, and L. S. Hultgren for numerous helpful discussions and suggestions.

\section{References}

1. J. W. MiLES, On the disturbed motion of a plane vortex sheet, J. Fluid Mech. 4:538-552 (1958).

2. M. ARtola and A. J. MAJDA, Nonlinear development of instabilities in supersonic vortex sheets, Physica D 28:253-281 (1987).

3. J. A. Pedelty and P. R. WoOdWARd, Numerical simulations of the nonlinear kink modes in linearly stable supersonic slip surfaces, J. Fluid Mech. 225:101-120 (1991).

4. G. M. BASSETT and P. R. WoOdWARD, Numerical simulation of nonlinear kink instabilities on supersonic shear layers, J. Fluid Mech. 284:323-340 (1995).

5. W. Blumen, P. G. Drazin, and D. F. Billings, Shear layer instability of an inviscid compressible fluid, 2, J. Fluid Mech. 71:305-316 (1975).

6. T. F. BALSA, Low-Frequency Nonlinear Modes in Supersonic Mixing Layers, American Physical Society Division of Fluid Dynamics Meeting, 1990.

7. T. F. BALSA, The stability of a supersonic mixing layer: Two-dimensional modes with nonlinear critical layers, 1996 [In preparation].

8. A. F. Messiter, Nonlinear Instability of Supersonic Vortex Sheets and Shear Layers, International Workshop on Advances in Analytical Methods in Aerodynamics, Miedzyzdroje, Poland, 1993.

9. J. Kevorkian and J. D. Cole, Perturbation Methods in Applied Mathematics, Springer, 1981.

10. P. Huerre and P. A. Monkewitz, Local and global instabilities in spatially developing flows, Ann. Rev. Fluid Mech. 22:473-537 (1990).

11. M. D. VAN DYKe, A Study of Second-Order Supersonic-Flow Theory, N. A. C. A. Rep. No. $1081,1952$. 
12. D. J. BENNEY and R. F. BERGERON, JR., A new class of nonlinear waves in parallel flows, Stud. Appl. Math. 48:181-204 (1969).

13. R. Haberman, Critical layers in parallel flows, Stud. Appl. Math. 51:139-161 (1972).

14. M. E. GOLDSTEIN and L. S. HULTGREN, Nonlinear spatial evolution of an externally excited instability wave in a free shear layer, J. Fluid Mech. 197:295-330 (1988).

15. S. N. Brown and K. Stewartson, The evolution of the critical layer of a Rossby wave, II, Geophys. Astrophys. Fluid Dynam. 10:1-24 (1978).

16. C. C. Lin, The Theory of Hydrodynamic Stability, Cambridge Univ. Press, 1955.

17. P. A. Lagerstrom, Matched Asymptotic Expansions, Springer, 1988.

18. S. M. Churilov and I. G. ShUKman, Note on weakly nonlinear stability of a free mixing layer, Proc. Roy. Soc. A 409:351-367 (1987).

UNIVERSITY OF MICHIGAN

(Received June 19, 1995) 